\title{
Entropy numbers of compact embeddings of Smoothness Morrey spaces on bounded domains
}

\author{
Dorothee D. Haroske, and Leszek Skrzypczak ${ }^{\dagger \dagger}$
}

April 22, 2020

\begin{abstract}
We study the compact embedding between smoothness Morrey spaces on bounded domains and characterise its entropy numbers. Here we discover a new phenomenon when the difference of smoothness parameters in the source and target spaces is rather small compared with the influence of the fine parameters in the Morrey setting. In view of some partial forerunners this was not to be expected till now. Our argument relies on wavelet decomposition techniques of the function spaces and a careful study of the related sequence space setting.
\end{abstract}

\section{Introduction}

Let $\Omega \subset \mathbb{R}^{d}$ be a bounded $C^{\infty}$ domain and $\mathcal{N}_{u, p, q}^{s}(\Omega)$ and $\mathcal{E}_{u, p, q}^{s}(\Omega)$ smoothness Morrey spaces, with $s_{i} \in \mathbb{R}, 0<p_{i} \leq u_{i}<\infty, 0<q_{i} \leq \infty, i=1,2$. Roughly speaking, these spaces $\mathcal{N}_{u, p, q}^{s}$ and $\mathcal{E}_{u, p, q}^{s}$ are the counterparts of the well-known Besov and Triebel-Lizorkin function spaces $B_{p, q}^{s}$ and $F_{p, q}^{s}$, respectively, where the basic $L_{p}$ space in the latter scales is replaced by the Morrey space $\mathcal{M}_{u, p}, 0<p \leq u<\infty$ : this is the set of all locally $p$-integrable functions $f \in L_{p}^{\text {loc }}\left(\mathbb{R}^{d}\right)$ such that

$$
\left\|f \mid \mathcal{M}_{u, p}\left(\mathbb{R}^{d}\right)\right\|=\sup _{x \in \mathbb{R}^{d}, R>0} R^{\frac{d}{u}-\frac{d}{p}}\left[\int_{B(x, R)}|f(y)|^{p} \mathrm{~d} y\right]^{\frac{1}{p}}<\infty .
$$

For the precise definition and further properties we refer to Section 2.1 below.

In our recent papers [13,14] we obtained a complete characterisation when the corresponding embeddings

$$
\operatorname{id}_{\mathcal{N}}: \mathcal{N}_{u_{1}, p_{1}, q_{1}}^{s_{1}}(\Omega) \rightarrow \mathcal{N}_{u_{2}, p_{2}, q_{2}}^{s_{2}}(\Omega) \quad \text { and } \quad \operatorname{id}_{\mathcal{E}}: \mathcal{E}_{u_{1}, p_{1}, q_{1}}^{s_{1}}(\Omega) \rightarrow \mathcal{E}_{u_{2}, p_{2}, q_{2}}^{s_{2}}(\Omega)
$$

are compact. The main purpose of the present paper is to characterise this compactness in terms of the corresponding entropy numbers. Apart from our first outcome in [16] (and some parallel results for approximation numbers in [1,50]) we are not aware of any other such investigation. This seems a little surprising in view of the popularity of smoothness Morrey spaces recently and the possible applications of entropy (and approximation) number results. However, these latter options are out of the scope of the present paper.

Smoothness Morrey spaces have been studied intensely in the past couple of years opening a wide field of possible applications. The Besov-Morrey spaces $\mathcal{N}_{u, p, q}^{s}\left(\mathbb{R}^{d}\right)$ were introduced in [19] by Kozono and Yamazaki and used by them and Mazzucato [26 to study Navier-Stokes equations. Corresponding Triebel-Lizorkin-Morrey spaces $\mathcal{E}_{u, p, q}^{s}\left(\mathbb{R}^{d}\right)$ were introduced in 41 by Tang and $\mathrm{Xu}$, where the authors established the Morrey version of Fefferman-Stein vectorvalued inequality. Properties of these spaces such as wavelet characterisations were studied in the papers by Sawano [33, 34], Sawano and Tanaka [36, 37], and Rosenthal [32]. We give some further references in Section 2.1 below.

\footnotetext{
*Institute of Mathematics, Friedrich Schiller University Jena, 07737 Jena, Germany

${ }^{\dagger}$ Faculty of Mathematics and Computer Science, Adam Mickiewicz University, 61-614 Poznan, Poland

$\ddagger$ The author was supported by National Science Center, Poland, Grant No.2013/10/A/ST1/00091.
} 
Now we concentrate on embeddings within the scale of such spaces. Let $s_{i} \in \mathbb{R}$, $0<p_{i} \leq u_{i}<\infty, 0<q_{i} \leq \infty, i=1,2$. In [12, 14 we characterised the continuity of the embeddings id $: \mathcal{N}_{u_{1}, p_{1}, q_{1}}^{s_{1}}\left(\mathbb{R}^{\bar{d}}\right) \rightarrow \mathcal{N}_{u_{2}, p_{2}, q_{2}}^{s_{2}}\left(\mathbb{R}^{d}\right)$ and id $: \mathcal{E}_{u_{1}, p_{1}, q_{1}}^{s_{1}}\left(\mathbb{R}^{d}\right) \rightarrow \mathcal{E}_{u_{2}, p_{2}, q_{2}}^{s_{2}}\left(\mathbb{R}^{d}\right)$. Furthermore, in [12 14 we studied some limiting embeddings. But these embeddings can never be compact. However, turning to spaces on bounded domains, we obtained in [13, 14] necessary and sufficient conditions for the corresponding embeddings $[1.2$ to be compact: this is the case if, and only if,

$$
\frac{s_{1}-s_{2}}{d}>\max \left\{0, \frac{1}{u_{1}}-\frac{1}{u_{2}}, \frac{p_{1}}{u_{1}}\left(\frac{1}{p_{1}}-\frac{1}{p_{2}}\right)\right\} \text {. }
$$

Recall that in case of $p_{i}=u_{i}, i=1,2$, we return to the classical situation of Besov and TriebelLizorkin spaces. Then the above findings (1.3) are in perfect agreement with the well-known condition $\frac{s_{1}-s_{2}}{d}>\max \left\{0, \frac{1}{p_{1}}-\frac{1}{p_{2}}\right\}$, for the compactness of $\operatorname{id}_{B}: B_{p_{1}, q_{1}}^{s_{1}}(\Omega) \rightarrow B_{p_{2}, q_{2}}^{s_{2}}(\Omega)$, similarly for $\operatorname{id}_{F}: F_{p_{1}, q_{1}}^{s_{1}}(\Omega) \rightarrow F_{p_{2}, q_{2}}^{s_{2}}(\Omega)$. However, a lot more about the compactness of the embeddings $\operatorname{id}_{B}, \operatorname{id}_{F}$ has been found in that 'classical' situation. The 'degree of compactness' as reflected by the asymptotic behaviour of the corresponding entropy numbers, is well-known; for the definition of entropy numbers as well as further applications of this concept we refer to Sec-

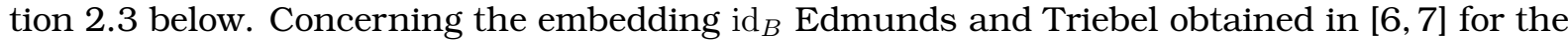
asymptotic decay of the entropy numbers that

$$
e_{k}\left(\operatorname{id}_{B}: B_{p_{1}, q_{1}}^{s_{1}}(\Omega) \rightarrow B_{p_{2}, q_{2}}^{s_{2}}(\Omega)\right) \quad \sim \quad k^{-\frac{s_{1}-s_{2}}{d}}, \quad k \in \mathbb{N},
$$

the result for $F$-spaces is parallel.

Now we tackle the Morrey situation in full generality, that is, we study the asymptotic behaviour of the entropy numbers of the compact embeddings $\mathrm{id}_{\mathcal{N}}$ and $\mathrm{id}_{\mathcal{E}}$ given by [1.2] whenever [1.3) is satisfied. In our recent contribution [16] we obtained first results in some cases, using sharp embeddings and interpolation techniques as main tools. In particular, under the additional assumption $\frac{s_{1}-s_{2}}{d}>\max \left\{0, \frac{1}{p_{1}}-\frac{1}{u_{2}}\right\}$ we could prove the same asymptotic behaviour of the entropy numbers of $\operatorname{id}_{\mathcal{N}}$ as in (1.4), with the observation, that the lower estimate remains valid in all cases of compactness $\left[1.3\right.$. Again, id $\mathrm{N}_{\mathcal{N}}$ can be replaced by id $\mathrm{E}_{\mathcal{E}}$ At this point the remaining case was left open, that is, when

$$
\max \left\{0, \frac{1}{u_{1}}-\frac{1}{u_{2}}, \frac{p_{1}}{u_{1}}\left(\frac{1}{p_{1}}-\frac{1}{p_{2}}\right)\right\}<\frac{s_{1}-s_{2}}{d} \leq \max \left\{0, \frac{1}{p_{1}}-\frac{1}{u_{2}}\right\}
$$

is satisfied. Plainly, this case requires at least the source space to be of 'proper' Morrey type, that is, $p_{1}<u_{1}$. Otherwise it would be excluded. Based on the 'classical' outcome [1.4), where always the difference of smoothness parameters $s_{1}-s_{2}$ characterises the decay of the entropy numbers, as well as the observation that our first approach in [16] verified the lower bound of that type always to hold, some reasonable assumption was that 1.4 should be extended to all cases admitted by (1.3). However, our present results disprove this claim: we found that the splitting point for the asymptotic behaviour of entropy numbers is $s_{1}-s_{2}=d\left(\frac{1}{p_{1}}-\frac{1}{p_{2}}\right)$, which is particularly remarkable since the $p_{i}$-parameters can be regarded as the fine tuning of the local integrability of the function $f$, recall 1.1 . So the interplay between smoothness and local regularity seems more important than expected so far, since in the local-global result (1.4) this difference was hidden. Our main outcome is the following:

(a) If $\frac{s_{1}-s_{2}}{d}>\max \left\{0, \frac{1}{u_{1}}-\frac{1}{u_{2}}, \frac{p_{1}}{u_{1}}\left(\frac{1}{p_{1}}-\frac{1}{p_{2}}\right)\right\}=\max \left\{0, \frac{1}{u_{1}}-\frac{1}{u_{2}}\right\}$, or $\frac{s_{1}-s_{2}}{d}>\frac{p_{1}}{u_{1}}\left(\frac{1}{p_{1}}-\frac{1}{p_{2}}\right)>$ $\max \left\{0, \frac{1}{u_{1}}-\frac{1}{u_{2}}\right\} \quad$ and $\quad \frac{s_{1}-s_{2}}{d}>\frac{1}{p_{1}}-\frac{1}{p_{2}}$, then

$$
e_{k}\left(\operatorname{id}_{\mathcal{N}}: \mathcal{N}_{u_{1}, p_{1}, q_{1}}^{s_{1}}(\Omega) \hookrightarrow \mathcal{N}_{u_{2}, p_{2}, q_{2}}^{s_{2}}(\Omega)\right) \sim k^{-\frac{s_{1}-s_{2}}{d}}, \quad k \in \mathbb{N} .
$$

(b) If $\max \left\{0, \frac{1}{u_{1}}-\frac{1}{u_{2}}\right\}<\frac{p_{1}}{u_{1}}\left(\frac{1}{p_{1}}-\frac{1}{p_{2}}\right)<\frac{s_{1}-s_{2}}{d} \leq \frac{1}{p_{1}}-\frac{1}{p_{2}}$, then there exists some $c>0$ and for any $\varepsilon>0$ some $c_{\varepsilon}>0$ such that for all $k \in \mathbb{N}$,

$$
c k^{-\alpha} \leq e_{k}\left(\operatorname{id}_{\mathcal{N}}\right) \leq c_{\varepsilon} k^{-\alpha+\varepsilon}
$$

with $\alpha=\frac{u_{1}}{u_{1}-p_{1}}\left(\frac{s_{1}-s_{2}}{d}-\frac{p_{1}}{u_{1}}\left(\frac{1}{p_{1}}-\frac{1}{p_{2}}\right)\right)$. 
Despite the (only) two-sided estimate in (1.6) the lower estimate already disproves the idea that [1.5] could be true in all cases. This is in our opinion the really astonishing part of the outcome, but also the other cases (beyond the one dealt with in [16]) are new. Moreover we prove that the above estimates hold for the smoothness Morrey spaces defined on an arbitrary bounded domain in $\mathbb{R}^{d}$.

The paper is organised as follows. In Section 2 we briefly introduce the corresponding function spaces and the concept of entropy numbers, in Section 3 the main results are proved. This is done in terms of appropriate sequence spaces which result from the decomposition of the function spaces by wavelets. In the concluding Section 4 we establish the results for function spaces and discuss some special situations.

\section{Preliminaries}

First we fix some notation. By $\mathbb{N}$ we denote the set of natural numbers, by $\mathbb{N}_{0}$ the set $\mathbb{N} \cup\{0\}$. For $a \in \mathbb{R}$, let $a_{+}:=\max \{a, 0\}$ and $\lfloor a\rfloor=\max \{k \in \mathbb{Z}: k \leq a\}$. For a set $\Omega \subset \mathbb{R}^{d}$ we denote its characteristic function by $\chi_{\Omega}$.

All unimportant positive constants will be denoted by $c$, occasionally with subscripts. The notation $A \lesssim B$ means that there exists a positive constant $c$ such that $A \leq c B$, whereas the symbol $A \sim B$ stands for $A \lesssim B \lesssim A$.

Given two (quasi-)Banach spaces $X$ and $Y$, we write $X \hookrightarrow Y$ if $X \subset Y$ and the natural embedding of $X$ into $Y$ is continuous.

\subsection{Smoothness Morrey spaces on $\mathbb{R}^{d}$}

Recall that the Morrey space $\mathcal{M}_{u, p}\left(\mathbb{R}^{d}\right), 0<p \leq u<\infty$, is defined to be the set of all locally $p$-integrable functions $f \in L_{p}^{\text {loc }}\left(\mathbb{R}^{d}\right)$ satisfying [1.1.

Remark 2.1. The spaces $\mathcal{M}_{u, p}\left(\mathbb{R}^{d}\right)$ are quasi-Banach spaces (Banach spaces for $p \geq 1$ ). They originated from Morrey's study on PDE (see [27]) and are part of the wider class of MorreyCampanato spaces; cf. [28]. They can be considered as a complement to $L_{p}$ spaces, since $\mathcal{M}_{p, p}\left(\mathbb{R}^{d}\right)=L_{p}\left(\mathbb{R}^{d}\right)$ with $p \in(0, \infty)$, extended by $\mathcal{M}_{\infty, \infty}\left(\mathbb{R}^{d}\right)=L_{\infty}\left(\mathbb{R}^{d}\right)$. In a parallel way one can define the spaces $\mathcal{M}_{\infty, p}\left(\mathbb{R}^{d}\right), p \in(0, \infty)$, but using the Lebesgue differentiation theorem, one arrives at $\mathcal{M}_{\infty, p}\left(\mathbb{R}^{d}\right)=L_{\infty}\left(\mathbb{R}^{d}\right)$. Moreover, $\mathcal{M}_{u, p}\left(\mathbb{R}^{d}\right)=\{0\}$ for $u<p$, and for $0<p_{2} \leq p_{1} \leq u<\infty$, $L_{u}\left(\mathbb{R}^{d}\right)=\mathcal{M}_{u, u}\left(\mathbb{R}^{d}\right) \hookrightarrow \mathcal{M}_{u, p_{1}}\left(\mathbb{R}^{d}\right) \hookrightarrow \mathcal{M}_{u, p_{2}}\left(\mathbb{R}^{d}\right)$.

Now we present the smoothness spaces of Morrey type in which we are interested. The Schwartz space $\mathcal{S}\left(\mathbb{R}^{d}\right)$ and its dual $\mathcal{S}^{\prime}\left(\mathbb{R}^{d}\right)$ of all complex-valued tempered distributions have their usual meaning here. Let $\varphi_{0}=\varphi \in \mathcal{S}\left(\mathbb{R}^{d}\right)$ be such that $\operatorname{supp} \varphi \subset\left\{y \in \mathbb{R}^{d}:|y|<2\right\}$ and $\varphi(x)=1$ if $|x| \leq 1$, and for each $j \in \mathbb{N}$ let $\varphi_{j}(x)=\varphi\left(2^{-j} x\right)-\varphi\left(2^{-j+1} x\right)$. Then $\left\{\varphi_{j}\right\}_{j=0}^{\infty}$ forms a smooth dyadic resolution of unity. Given any $f \in \mathcal{S}^{\prime}\left(\mathbb{R}^{d}\right)$, we denote by $\mathcal{F} f$ and $\mathcal{F}^{-1} f$ its Fourier transform and its inverse Fourier transform, respectively.

Definition 2.2. Let $0<p \leq u<\infty$ or $p=u=\infty$. Let $0<q \leq \infty, s \in \mathbb{R}$ and $\left\{\varphi_{j}\right\}_{j}$ a smooth dyadic resolution of unity.

(i) The Besov-Morrey space $\mathcal{N}_{u, p, q}^{s}\left(\mathbb{R}^{d}\right)$ is defined to be the set of all distributions $f \in \mathcal{S}^{\prime}\left(\mathbb{R}^{d}\right)$ such that

$$
\left\|f \mid \mathcal{N}_{u, p, q}^{s}\left(\mathbb{R}^{d}\right)\right\|:=\left[\sum_{j=0}^{\infty} 2^{j s q}\left\|\mathcal{F}^{-1}\left(\varphi_{j} \mathcal{F} f\right) \mid \mathcal{M}_{u, p}\left(\mathbb{R}^{d}\right)\right\|^{q}\right]^{1 / q}<\infty
$$

with the usual modification made in case of $q=\infty$.

(ii) Let $u \in(0, \infty)$. The Triebel-Lizorkin-Morrey space $\mathcal{E}_{u, p, q}^{s}\left(\mathbb{R}^{d}\right)$ is defined to be the set of all distributions $f \in \mathcal{S}^{\prime}\left(\mathbb{R}^{d}\right)$ such that

$$
\left\|f\left|\mathcal{E}_{u, p, q}^{s}\left(\mathbb{R}^{d}\right)\|:=\|\left[\sum_{j=0}^{\infty} 2^{j s q}\left|\mathcal{F}^{-1}\left(\varphi_{j} \mathcal{F} f\right)\right|^{q}\right]^{1 / q}\right| \mathcal{M}_{u, p}\left(\mathbb{R}^{d}\right)\right\|<\infty
$$

with the usual modification made in case of $q=\infty$. 
Convention. We adopt the nowadays usual custom to write $A_{p, q}^{s}$ instead of $B_{p, q}^{s}$ or $F_{p, q}^{s}$, and $\mathcal{A}_{u, p, q}^{s}$ instead of $\mathcal{N}_{u, p, q}^{s}$ or $\mathcal{E}_{u, p, q}^{s}$, respectively, when both scales of spaces are meant simultaneously in some context.

Remark 2.3. The spaces $\mathcal{A}_{u, p, q}^{s}\left(\mathbb{R}^{d}\right)$ are independent of the particular choice of the smooth dyadic resolution of unity $\left\{\varphi_{j}\right\}_{j}$ appearing in their definitions. They are quasi-Banach spaces (Banach spaces for $p, q \geq 1$ ), and $\mathcal{S}\left(\mathbb{R}^{d}\right) \hookrightarrow \mathcal{A}_{u, p, q}^{s}\left(\mathbb{R}^{d}\right) \hookrightarrow \mathcal{S}^{\prime}\left(\mathbb{R}^{d}\right)$. When $u=p$, they coincide with the usual Besov and Triebel-Lizorkin spaces, $\mathcal{A}_{p, p, q}^{s}\left(\mathbb{R}^{d}\right)=A_{p, q}^{s}\left(\mathbb{R}^{d}\right)$. There exists extensive literature on the latter spaces; we refer, in particular, to the series of monographs [43,44,46] for a comprehensive treatment. In case of $u<p$ we have $\mathcal{A}_{u, p, q}^{s}\left(\mathbb{R}^{d}\right)=\{0\}$. We occasionally benefit from the elementary embeddings

$$
B_{p, \min \{p, q\}}^{s}\left(\mathbb{R}^{d}\right) \hookrightarrow F_{p, q}^{s}\left(\mathbb{R}^{d}\right) \hookrightarrow B_{p, \max \{p, q\}}^{s}\left(\mathbb{R}^{d}\right),
$$

where $p \in(0, \infty), q \in(0, \infty]$ and $s \in \mathbb{R}$. The result for spaces $\mathcal{A}_{u, p, q}^{s}$ is different: Sawano proved in 34 that, for $s \in \mathbb{R}$ and $0<p<u<\infty$,

$$
\mathcal{N}_{u, p, \min \{p, q\}}^{s}\left(\mathbb{R}^{d}\right) \hookrightarrow \mathcal{E}_{u, p, q}^{s}\left(\mathbb{R}^{d}\right) \hookrightarrow \mathcal{N}_{u, p, \infty}^{s}\left(\mathbb{R}^{d}\right),
$$

where, for the latter embedding, $r=\infty$ cannot be improved. Mazzucato has shown in [26, Prop. 4.1] that $\mathcal{E}_{u, p, 2}^{0}\left(\mathbb{R}^{d}\right)=\mathcal{M}_{u, p}\left(\mathbb{R}^{d}\right), \quad 1<p \leq u<\infty$.

Remark 2.4. Other important, closely related scales of spaces are the so-called Besov-type spaces $B_{p, q}^{s, \tau}\left(\mathbb{R}^{d}\right)$ and Triebel-Lizorkin-type spaces $F_{p, q}^{s, \tau}\left(\mathbb{R}^{d}\right)$, introduced in [51], as well as the local and hybrid spaces dealt with in [47] and [48], cf. also [39,40] and [49,50].

We briefly recall the wavelet characterisation of Besov-Morrey spaces proved in [34]. It will be essential for our final results and motivates our studies on sequence spaces. For $m \in \mathbb{Z}^{d}$ and $\nu \in \mathbb{Z}$ we define a $d$-dimensional dyadic cube with sides parallel to the axes of coordinates by $Q_{\nu, m}=\prod_{i=1}^{d}\left[\frac{m_{i}}{2^{\nu}}, \frac{m_{i}+1}{2^{\nu}}\right), \nu \in \mathbb{Z}, m=\left(m_{1}, \ldots, m_{d}\right) \in \mathbb{Z}^{d}$. For $0<u<\infty, \nu \in \mathbb{Z}$ and $m \in \mathbb{Z}^{d}$ we denote by $\chi_{\nu, m}^{(u)}$ the $u$-normalised characteristic function of the cube $Q_{\nu, m}, \quad \chi_{\nu, m}^{(u)}=2^{\nu d / u} \chi_{Q_{\nu, m}}$, hence $\left\|\chi_{\nu, m}^{(u)} \mid L_{p}\right\|=1$ and $\left\|\chi_{\nu, m}^{(u)} \mid \mathcal{M}_{u, p}\right\|=1$.

Let $\widetilde{\phi}$ be a scaling function on $\mathbb{R}$ with compact support and of sufficiently high regularity. Let $\widetilde{\psi}$ be an associated wavelet. Then the tensor-product ansatz yields a scaling function $\phi$ and associated wavelets $\psi_{1}, \ldots, \psi_{2^{d}-1}$, all defined now on $\mathbb{R}^{d}$. We suppose $\widetilde{\phi} \in C^{N_{1}}(\mathbb{R})$ and $\operatorname{supp} \widetilde{\phi} \subset\left[-N_{2}, N_{2}\right]$ for certain natural numbers $N_{1}$ and $N_{2}$. This implies

$$
\phi, \psi_{i} \in C^{N_{1}}\left(\mathbb{R}^{d}\right) \text { and } \operatorname{supp} \phi, \operatorname{supp} \psi_{i} \subset\left[-N_{3}, N_{3}\right]^{d},
$$

for $i=1, \ldots, 2^{d}-1$. We use the standard abbreviations

$$
\phi_{\nu, m}(x)=2^{\nu d / 2} \phi\left(2^{\nu} x-m\right) \text { and } \psi_{i, \nu, m}(x)=2^{\nu d / 2} \psi_{i}\left(2^{\nu} x-m\right) .
$$

To formulate the result we introduce some sequence spaces. For $0<p \leq u<\infty, 0<q \leq \infty$ and $\sigma \in \mathbb{R}$, let

$$
\begin{aligned}
n_{u, p, q}^{\sigma}:=\left\{\lambda=\left\{\lambda_{\nu, m}\right\}_{\nu, m}:\right. & \lambda_{\nu, m} \in \mathbb{C}, \\
& \left.\left\|\lambda\left|n_{u, p, q}^{\sigma}\|=\|\left\{2^{\nu\left(\sigma-\frac{d}{u}\right)}\left\|\sum_{m \in \mathbb{Z}^{d}} \lambda_{\nu, m} \chi_{\nu, m}^{(u)} \mid \mathcal{M}_{u, p}\right\|\right\}_{\nu \in \mathbb{N}_{0}}\right| \ell_{q}\right\|<\infty\right\} .
\end{aligned}
$$

The following theorem was proved in [34].

Theorem 2.5. Let $0<p \leq u<\infty$ or $u=p=\infty, 0<q \leq \infty$ and let $s \in \mathbb{R}$. Let $\phi$ be a scaling function and let $\psi_{i}, i=1, \ldots, 2^{d}-1$, be the corresponding wavelets satisfying (2.3). We assume that $\max \left\{(1+\lfloor s\rfloor)_{+},\left\lfloor d\left(\frac{1}{p}-1\right)_{+}-s\right\rfloor\right\} \leq N_{1}$. Then a distribution $f \in \mathcal{S}^{\prime}\left(\mathbb{R}^{d}\right)$ belongs to $\mathcal{N}_{u, p, q}^{s}\left(\mathbb{R}^{d}\right)$, if, and only if,

$$
\left\|f\left|\mathcal{N}_{u, p, q}^{s}\left(\mathbb{R}^{d}\right)\left\|^{\star}=\right\|\left\{\left\langle f, \phi_{0, m}\right\rangle\right\}_{m \in \mathbb{Z}^{d}}\right| \ell_{u}\right\|+\sum_{i=1}^{2^{d}-1}\left\|\left\{\left\langle f, \psi_{i, \nu, m}\right\rangle\right\}_{\nu \in \mathbb{N}_{0}, m \in \mathbb{Z}^{d}} \mid n_{u, p, q}^{\sigma}\right\|
$$

is finite, where $\sigma=s+\frac{d}{2}$. Furthermore, $\left\|f \mid \mathcal{N}_{u, p, q}^{s}\left(\mathbb{R}^{d}\right)\right\|^{\star}$ may be used as an equivalent (quasi-) norm in $\mathcal{N}_{u, p, q}^{s}\left(\mathbb{R}^{d}\right)$. 
Remark 2.6. It follows from Theorem 2.5 that the mapping

$$
T: f \mapsto\left(\left\{\left\langle f, \phi_{0, m}\right\rangle\right\}_{m \in \mathbb{Z}^{d}},\left\{\left\langle f, \psi_{i, \nu, m}\right\rangle\right\}_{\nu \in \mathbb{N}_{0}, m \in \mathbb{Z}^{d}, i=1, \ldots, 2^{d}-1}\right)
$$

is an isomorphism of $\mathcal{N}_{u, p, q}^{s}\left(\mathbb{R}^{d}\right)$ onto $\ell_{u} \oplus\left(\oplus_{i=1}^{2^{d}-1} n_{u, p, q}^{\sigma}\right), \sigma=s+\frac{d}{2}$, cf. [34].

The theorem covers the characterisation of Besov spaces $B_{p, q}^{s}\left(\mathbb{R}^{d}\right)$ by Daubechies wavelets, cf. [46, p.26-34] and the references given there.

Remark 2.7. In [12] we defined an equivalent norm in the sequence spaces $n_{u, p, q}^{\sigma}$ that is more convenient for our purposes. Let $\sigma \in \mathbb{R}, 0<q \leq \infty, 0<p \leq u<\infty$ or $u=p=\infty$. For a sequence $\left\{\lambda_{j, m}\right\}_{j, m}, j \in \mathbb{N}_{0}, m \in \mathbb{Z}^{d}$, consider a quasi-norm

$$
\left\|\lambda \mid n_{u, p, q}^{\sigma}\right\|^{*}=\left(\sum_{j=0}^{\infty} 2^{q j\left(\sigma-\frac{d}{u}\right)} \sup _{\nu: \nu \leq j ; k \in \mathbb{Z}^{d}} 2^{q d(j-\nu)\left(\frac{1}{u}-\frac{1}{p}\right)}\left(\sum_{m: Q_{j, m} \subset Q_{\nu, k}}\left|\lambda_{j, m}\right|^{p}\right)^{\frac{q}{p}}\right)^{\frac{1}{q}},
$$

with the usual modification if $q=\infty$ or $u=p=\infty$. Then

$$
n_{u, p, q}^{\sigma}=\left\{\lambda=\left\{\lambda_{j, m}\right\}_{j, m}: j \in \mathbb{N}_{0}, \quad m \in \mathbb{Z}^{d} \text { and }\left\|\lambda \mid n_{u, p, q}^{\sigma}\right\|^{*}<\infty\right\} .
$$

\subsection{Function spaces on domains}

We assume that $\Omega$ is a bounded open set in $\mathbb{R}^{d}$. We consider smoothness Morrey spaces on $\Omega$ defined by restriction.

Definition 2.8. Let $0<p \leq u<\infty$ or $p=u=\infty, q \in(0, \infty]$ and $s \in \mathbb{R}$ (with $u<\infty$ in the case of $\mathcal{A}=\mathcal{E})$. Then $\mathcal{A}_{u, p, q}^{s}(\Omega)$ is defined by

$$
\mathcal{A}_{u, p, q}^{s}(\Omega):=\left\{f \in \mathcal{D}^{\prime}(\Omega): f=\left.g\right|_{\Omega} \text { for some } g \in \mathcal{A}_{u, p, q}^{s}\left(\mathbb{R}^{d}\right)\right\}
$$

endowed with the quasi-norm

$$
\left\|f \mid \mathcal{A}_{u, p, q}^{s}(\Omega)\right\|:=\inf \left\{\left\|g\left|\mathcal{A}_{u, p, q}^{s}\left(\mathbb{R}^{d}\right) \|: f=g\right|_{\Omega}, g \in \mathcal{A}_{u, p, q}^{s}\left(\mathbb{R}^{d}\right)\right\} .\right.
$$

Remark 2.9. The spaces $\mathcal{A}_{u, p, q}^{s}(\Omega)$ are quasi-Banach spaces (Banach spaces for $p, q \geq 1$ ). Several properties of the spaces $\mathcal{A}_{u, p, q}^{s}(\Omega)$, including the extension property, were studied in [35] in the case when $\Omega$ is a bounded $C^{\infty}$ domain in $\mathbb{R}^{d}$. With the same assumption, embeddings within spaces in this latter scale as well as to classical spaces like $C(\Omega)$ or $L_{r}(\Omega)$ were investigated in [13, 14]. In [9] we studied the question under what assumptions these spaces consist of regular distributions only.

We recall in detail our compactness result as obtained in [13] (for $\mathcal{A}=\mathcal{N}$ ) and [14] (for $\mathcal{A}=\mathcal{E})$.

Theorem 2.10. Let $\Omega$ be a bounded $C^{\infty}$ domain in $\mathbb{R}^{d}, s_{i} \in \mathbb{R}, 0<q_{i} \leq \infty, 0<p_{i} \leq u_{i}<\infty$, $i=1,2$. Then the embedding

$$
\operatorname{id}_{\mathcal{A}}: \mathcal{A}_{u_{1}, p_{1}, q_{1}}^{s_{1}}(\Omega) \hookrightarrow \mathcal{A}_{u_{2}, p_{2}, q_{2}}^{s_{2}}(\Omega)
$$

is compact if, and only if, the following condition holds

$$
\frac{s_{1}-s_{2}}{d}>\max \left\{0, \frac{1}{u_{1}}-\frac{1}{u_{2}}, \frac{p_{1}}{u_{1}}\left(\frac{1}{p_{1}}-\frac{1}{p_{2}}\right)\right\} .
$$

In case of the target space $L_{\infty}(\Omega)$ we obtained in [13, 14] the following result, again for bounded $C^{\infty}$ domains $\Omega$.

Proposition 2.11. Let $s \in \mathbb{R}, 0<p<u<\infty$ and $q \in(0, \infty]$. Then $\mathcal{A}_{u, p, q}^{s}(\Omega) \hookrightarrow L_{\infty}(\Omega)$ is compact, if, and only if, $s>\frac{d}{u}$.

Finally we state some outcome on real interpolation of Besov-Morrey spaces on $\Omega$ which will be of great use for us in the sequel. It can be proved similarly to the proof of [46, Thm. 1.110], relying on the corresponding assertions with $\Omega$ replaced by $\mathbb{R}^{d}$ that can be found in 40 , Thm. 2.2, Prop. 2.3], and on the existence of a common extension operator, cf. [35, Thm. 5.4]. 
Theorem 2.12. Let $u, q, q_{0}, q_{1} \in(0, \infty]$ and $\theta \in(0,1)$.

(i) Let $0<p \leq u<\infty$ and $s_{0}, s_{1} \in \mathbb{R}$ with $s_{0} \neq s_{1}$. Then

$$
\mathcal{N}_{u, p, q}^{s}(\Omega)=\left(\mathcal{N}_{u, p, q_{0}}^{s_{0}}(\Omega), \mathcal{N}_{u, p, q_{1}}^{s_{1}}(\Omega)\right)_{\theta, q} \quad \text { if } \quad s=(1-\theta) s_{0}+\theta s_{1} .
$$

(ii) Let $1 \leq p \leq u<\infty$ and $s \in \mathbb{R}$. Then

$$
\mathcal{N}_{u, p, q}^{s}(\Omega)=\left(\mathcal{N}_{u, p, q_{0}}^{s}(\Omega), \mathcal{N}_{u, p, q_{1}}^{s}(\Omega)\right)_{\theta, q} \quad \text { if } \quad \frac{1}{q}=\frac{1-\theta}{q_{0}}+\frac{\theta}{q_{1}} .
$$

\subsection{Entropy numbers}

As explained in the beginning already, our main concern in this paper is to characterise the compactness of embeddings in further detail. Therefore we briefly recall the concept of entropy numbers.

Definition 2.13. Let $X$ and $Y$ be two complex (quasi-) Banach spaces, $k \in \mathbb{N}$ and let $T \in$ $\mathcal{L}(X, Y)$ be a linear and continuous operator from $X$ into $Y$. The $k$ th entropy number $e_{k}(T)$ of $T$ is the infimum of all numbers $\varepsilon>0$ such that there exist $2^{k-1}$ balls in $Y$ of radius $\varepsilon$ which cover the image $T B_{X}$ of the unit ball $B_{X}=\{x \in X:\|x \mid X\| \leq 1\}$.

Remark 2.14. For details and properties of entropy numbers we refer to [3,4,18,30] (restricted to the case of Banach spaces), and [8] for some extensions to quasi-Banach spaces. Among other features we only want to mention the multiplicativity of entropy numbers: let $X, Y, Z$ be complex (quasi-) Banach spaces and $T_{1} \in \mathcal{L}(X, Y), T_{2} \in \mathcal{L}(Y, Z)$. Then

$$
e_{k_{1}+k_{2}-1}\left(T_{2} \circ T_{1}\right) \leq e_{k_{1}}\left(T_{1}\right) e_{k_{2}}\left(T_{2}\right), \quad k_{1}, k_{2} \in \mathbb{N} .
$$

Note that one has $\lim _{k \rightarrow \infty} e_{k}(T)=0$ if, and only if, $T$ is compact. The last equivalence justifies the saying that entropy numbers measure 'how compact' an operator acts. This is one reason to study the asymptotic behaviour of entropy numbers (that is, their decay) for compact operators in detail. Another one is the application to estimate eigenvalues; we refer to the monographs [3, 4, 8, 18, 30] for further details.

We recall the following property of entropy numbers which was obtained (in the Banach case situation) in [42, Sect. 1.16.2] and [29, 12.1] and extended to the quasi-Banach case setting in [17, Theorem 3.2] and [8, Theorem 1.3.2]. Let $A$ be a quasi-Banach space and let $\left\{B_{0}, B_{1}\right\}$ be an interpolation couple of quasi-Banach spaces. Let $0<\theta<1$ and let $B_{\theta}$ be a quasi-Banach space such that $B_{0} \cap B_{1} \hookrightarrow B_{\theta} \hookrightarrow B_{0}+B_{1}$ (naturally quasi-normed) and

$$
\left\|b\left|B_{\theta}\|\leq\| b\right| B_{0}\right\|^{1-\theta}\left\|b \mid B_{1}\right\|^{\theta} \quad \text { for all } \quad b \in B_{0} \cap B_{1} .
$$

Let $T \in \mathcal{L}\left(A, B_{0} \cap B_{1}\right)$. Then there is a number $c>0$ such that for all $k \in \mathbb{N}$,

$$
e_{2 k}\left(T: A \hookrightarrow B_{\theta}\right) \leq c e_{k}^{1-\theta}\left(T: A \hookrightarrow B_{0}\right) e_{k}^{\theta}\left(T: A \hookrightarrow B_{1}\right) .
$$

Now we benefit from the technique of quasi-normed operator ideals. In the context of entropy numbers the approach goes back to Carl [2]. It was used for Sobolev embeddings first time in [21,22]. For a bounded linear operator $T \in \mathcal{L}(X, Y)$, where $X$ and $Y$ are (quasi)-Banach spaces, and a positive real number $r$ we put

$$
L_{r, \infty}^{(e)}(P):=\sup _{n \in \mathbb{N}} n^{1 / r} e_{n}(P) .
$$

This is a quasi-norm (in general not a norm) for the operator ideal of all operators $P$ with $L_{r, \infty}^{(e)}(P)<\infty$, cf. Pietsch [29,30].

One of the main tools in our arguments will be the characterisation of the asymptotic behaviour of the entropy numbers of the embedding $\ell_{p_{1}}^{N} \hookrightarrow \ell_{p_{2}}^{N}$. For all $n \in \mathbb{N}$ we have

$$
e_{k}\left(\text { id }: \ell_{p_{1}}^{N} \hookrightarrow \ell_{p_{2}}^{N}\right) \sim \begin{cases}1 & \text { if } \quad 1 \leq k \leq \log 2 N, \\ \left(\frac{\log \left(1+\frac{N}{k}\right)}{k}\right)^{\frac{1}{p_{1}}-\frac{1}{p_{2}}} & \text { if } \quad \log 2 N \leq k \leq 2 N, \\ 2^{-\frac{k}{2 N} N^{\frac{1}{p_{2}}}-\frac{1}{p_{1}}} & \text { if } \quad 2 N \leq k\end{cases}
$$


and if $0<p_{2}<p_{1} \leq \infty$ it holds

$$
e_{k}\left(\mathrm{id}: \ell_{p_{1}}^{N} \hookrightarrow \ell_{p_{2}}^{N}\right) \sim 2^{-\frac{k}{2 N}} N^{\frac{1}{p_{2}}-\frac{1}{p_{1}}} \quad \text { for all } k \in \mathbb{N} .
$$

In the case $1 \leq p_{1}, p_{2} \leq \infty$ this has been proved by Schütt [38]. For $p_{1}<1$ and/or $p_{2}<1$ we refer to Edmunds and Triebel [8] and Triebel [45, 7.2, 7.3] (with a little supplement in [20]).

The characterisation of the asymptotic behaviour of the entropy numbers $e_{k}\left(\ell_{p_{1}}^{N} \hookrightarrow \ell_{p_{2}}^{N}\right)$, recall (2.15) and (2.16), implies

$$
L_{r, \infty}^{(e)}\left(\text { id }: \ell_{p_{1}}^{N} \hookrightarrow \ell_{p_{2}}^{N}\right) \sim N^{\frac{1}{r}-\frac{1}{p}} \quad \text { if } \frac{1}{r}>\max \left(0, \frac{1}{p}\right) \text { and } \frac{1}{p}=\frac{1}{p_{1}}-\frac{1}{p_{2}} .
$$

\section{Entropy numbers of compact embeddings in sequence spaces}

Recall our remarks about the wavelet characterisation of spaces $\mathcal{N}_{u, p, q}^{s}\left(\mathbb{R}^{d}\right)$, in particular, Theorem 2.5. Our strategy in estimating corresponding entropy numbers will be to transfer the question to the appropriate sequence spaces via wavelet decompositions. This method will be explained in further detail in the beginning of Section 4 below. We begin with the sequence space problem and have thus to adapt our sequence spaces to the spaces on bounded domains first.

Remark 3.1. Note that we use the most convenient setting for this purpose: we assume (implicitly) that the supports of the corresponding distributions are inside the domain, thus avoiding boundary wavelets. It turns out that this apparently simpler situation is already sufficient for our problem. This justifies our approach below. Note that we already used a similar argument in [13,14] when dealing with the compactness assertion.

Let $Q$ be a unit cube, $0<p \leq u<\infty, \sigma \in \mathbb{R}, 0<q \leq \infty$. We define a sequence space $\widetilde{n}_{u, p, q}^{\sigma}(Q)$ putting

$$
\widetilde{n}_{u, p, q}^{\sigma}(Q):=\left\{\lambda=\left\{\lambda_{j, m}\right\}_{j, m}: \lambda_{j, m} \in \mathbb{C}, Q_{j, m} \subset Q \text {, and }\left\|\lambda \mid \widetilde{n}_{u, p, q}^{\sigma}\right\|<\infty\right\},
$$

where

$$
\left\|\lambda \mid \widetilde{n}_{u, p, q}^{\sigma}\right\|=\left(\sum_{j=0}^{\infty} 2^{j q\left(\sigma-\frac{d}{u}\right)} \sup _{\nu: \nu \leq j ; k: Q_{\nu, k} \subset Q} 2^{q d(j-\nu)\left(\frac{1}{u}-\frac{1}{p}\right)}\left(\sum_{m: Q_{j, m} \subset Q_{\nu, k}}\left|\lambda_{j, m}\right|^{p}\right)^{\frac{q}{p}}\right)^{\frac{1}{q}},
$$

with the usual modification when $q=\infty$. Moreover for fixed $j \in \mathbb{N}_{0}$ we put

$$
m_{u, p}^{2^{j d}}=\left\{\lambda=\left\{\lambda_{j, m}\right\}_{j, m}: \quad \lambda_{j, m} \in \mathbb{C} \quad Q_{j, m} \subset Q \quad \text { and } \quad\left\|\lambda \mid m_{u, p}^{2^{j d}}\right\|<\infty\right\},
$$

where $\quad\left\|\lambda \mid m_{u, p}^{2^{j d}}\right\|=\sup _{\nu: \nu \leq j ; k \in \mathbb{Z}^{d}} 2^{d(j-\nu)\left(\frac{1}{u}-\frac{1}{p}\right)}\left(\sum_{m: Q_{j, m} \subset Q_{\nu, k} \subset Q}\left|\lambda_{j, m}\right|^{p}\right)^{\frac{1}{p}}$.

Remark 3.2. If $p=u$, then the spaces $\tilde{n}_{p, p, q}^{\sigma}(Q)=\tilde{b}_{p, q}^{\sigma}(Q)$ coincide with the spaces $\ell_{q}\left(2^{j\left(\sigma-\frac{d}{p}\right)} \ell_{p}^{M_{j}}\right)$, $M_{j} \sim 2^{j d}$, as dealt with in [45, Sect. 8.1]. Then in case of the (sequence space version of the) compact embedding (2.9) recalled in Theorem 2.10, that is, id : $\widetilde{n}_{u_{1}, p_{1}, q_{1}}^{\sigma_{1}} \hookrightarrow \widetilde{n}_{u_{2}, p_{2}, q_{2}}^{\sigma_{2}}$, it can always be extended to the chain of embeddings $\tilde{b}_{u_{1}, q_{1}}^{\sigma_{1}} \hookrightarrow \widetilde{n}_{u_{1}, p_{1}, q_{1}}^{\sigma_{1}} \hookrightarrow \widetilde{n}_{u_{2}, p_{2}, q_{2}}^{\sigma_{2}} \hookrightarrow \tilde{b}_{p_{2}, q_{2}}^{\sigma_{2}}$, where the first embedding is just by monotonicity and the last using Hölder's inequality and the boundedness of $Q$. Thus the multiplicativity of entropy numbers 2.11 immediately leads to

$$
e_{k}\left(\widetilde{n}_{u_{1}, p_{1}, q_{1}}^{\sigma_{1}} \hookrightarrow \widetilde{n}_{u_{2}, p_{2}, q_{2}}^{\sigma_{2}}\right) \geq c e_{k}\left(\tilde{b}_{u_{1}, q_{1}}^{\sigma_{1}} \hookrightarrow \tilde{b}_{p_{2}, q_{2}}^{\sigma_{2}}\right) \sim k^{-\frac{\sigma_{1}-\sigma_{2}}{d}}
$$

in all cases admitted by (2.10); the latter equivalence can be found in [45, Thm. 8.2].

Remark 3.3. It is obvious from Theorem 2.5 that the smoothness parameters $s$ and $\sigma$, appearing on the function spaces and sequence spaces side, respectively, are linked by $\sigma=s+\frac{d}{2}$, so in all cases below where the difference $\sigma_{1}-\sigma_{2}$ appears, we could equally call it $s_{1}-s_{2}$.

We collect some recent results from our paper [15] needed for our arguments below. 
Lemma 3.4 (cf. [15]). Let $0<p_{i} \leq u_{i}<\infty, i=1,2$, and $j \in \mathbb{N}_{0}$ be given. Then the norm of the compact identity operator

$$
\operatorname{id}_{j}: m_{u_{1}, p_{1}}^{2^{j d}} \hookrightarrow m_{u_{2}, p_{2}}^{2^{j d}}
$$

satisfies

$$
\left\|\operatorname{id}_{j}\right\|=\left\{\begin{array}{lll}
1 & \text { if } p_{1} \geq p_{2} \text { and } u_{2} \geq u_{1}, \\
1 & \text { if } p_{1}<p_{2} \text { and } \frac{p_{2}}{u_{2}} \leq \frac{p_{1}}{u_{1}} \\
2^{j d\left(\frac{1}{u_{2}}-\frac{1}{u_{1}}\right)} & \text { if } p_{1} \geq p_{2} \text { and } u_{2}<u_{1},
\end{array}\right.
$$

and in the remaining case, there is a constant $c, 0<c \leq 1$, independent of $j$ such that

$$
c 2^{j d\left(\frac{1}{u_{2}}-\frac{p_{1}}{u_{1} p_{2}}\right)} \leq\left\|\operatorname{id}_{j}\right\| \leq 2^{j d\left(\frac{1}{u_{2}}-\frac{p_{1}}{u_{1} p_{2}}\right)} \quad \text { if } \quad p_{1}<p_{2} \text { and } \frac{p_{2}}{u_{2}}>\frac{p_{1}}{u_{1}} .
$$

Using Schütt's result [2.15] and [2.16] together with some embedding arguments we obtained in [15] first entropy number estimates.

Lemma 3.5 (cf. [15]). Let $j \in \mathbb{N}, 0<p_{i} \leq u_{i}<\infty, i=1,2$, and $k \in \mathbb{N}_{0}$ with $k \gtrsim 2^{j d}$. Then

$$
e_{k}\left(\operatorname{id}_{j}: m_{u_{1}, p_{1}}^{2^{j d}} \rightarrow m_{u_{2}, p_{2}}^{2^{j d}}\right) \sim 2^{-k 2^{-j d}} 2^{j d\left(\frac{1}{u_{2}}-\frac{1}{u_{1}}\right)} .
$$

Now we are able to extend this in some sense. Recall that for a bounded subset $K$ of a finite-dimensional (quasi)-Banach space $Y$, the $k$-th entropy number $e_{k}(K, Y)$ is defined as

$$
\left.e_{k}(K, Y)=\min \left\{\varepsilon>0: K \subset \bigcup_{i=1}^{2^{k-1}}\left(x_{i}+\varepsilon B_{Y}\right)\right) \text { for some } x_{1}, \ldots x_{2^{k-1}} \in Y\right\} .
$$

If $X$ denotes the vector space $Y$ equipped with another (quasi)-norm, then $e_{k}\left(B_{X}, Y\right)=e_{k}($ id : $X \rightarrow Y)$. One can also easily check that $e_{k}(r K, Y)=r e_{k}(K, Y)$ if $r>0$.

Lemma 3.6. Let $j \in \mathbb{N}, 0<p_{2} \leq u_{2}<p_{1} \leq u_{1}<\infty$ and $k \in \mathbb{N}_{0}$. Then

$$
e_{k}\left(\operatorname{id}_{j}: m_{u_{1}, p_{1}}^{2^{j d}} \rightarrow m_{u_{2}, p_{2}}^{2^{j d}}\right) \sim 2^{-k 2^{-j d}} 2^{j d\left(\frac{1}{u_{2}}-\frac{1}{u_{1}}\right)} .
$$

Proof. Let $B_{q}(0, r), r>0$, denote the ball of radius $r$ centred at the origin in the space $\ell_{q}^{2^{j d}}$ and $B_{u, p}(0, r)$ the corresponding ball in the space $m_{u, p}^{2^{j d}}$. Directly from the definition of the norm in the space $m_{u_{1}, p_{1}}^{2^{j d}}$ it follows that

$$
B_{u}(0,1) \subset B_{u, p}(0,1) \subset B_{p}\left(0,2^{j d\left(\frac{1}{p}-\frac{1}{u}\right)}\right) .
$$

In consequence, if $q<p_{1} \leq u_{1}$, then

$$
e_{k}\left(B_{u_{1}, p_{1}}(0,1), \ell_{q}\right) \geq e_{k}\left(B_{u_{1}}(0,1), \ell_{q}^{2^{j d}}\right) \sim 2^{-k 2^{-j d}} 2^{j d\left(\frac{1}{q}-\frac{1}{u_{1}}\right)}
$$

and

$$
\begin{aligned}
e_{k}\left(B_{u_{1}, p_{1}}(0,1), \ell_{q}^{2^{j d}}\right) & \leq e_{k}\left(B_{p_{1}}\left(0,2^{j d\left(\frac{1}{p_{1}}-\frac{1}{u_{1}}\right)}\right), \ell_{q}^{2^{j d}}\right) \\
& =2^{j d\left(\frac{1}{p_{1}}-\frac{1}{u_{1}}\right)} e_{k}\left(B_{p_{1}}(0,1), \ell_{q}^{2^{j d}}\right) \\
& \sim 2^{-k 2^{-j d}} 2^{j d\left(\frac{1}{q}-\frac{1}{u_{1}}\right)} .
\end{aligned}
$$

Now, taking $q=u_{2}$ in 3.9, we obtain

$$
\begin{aligned}
e_{k}\left(\operatorname{id}_{j}: m_{u_{1}, p_{1}}^{2^{j d}} \rightarrow m_{u_{2}, p_{2}}^{2^{j d}}\right) & \leq e_{k}\left(\operatorname{id}_{j}: m_{u_{1}, p_{1}}^{2^{j d}} \rightarrow \ell_{u_{2}}^{2^{j d}}\right)\left\|\mathrm{id}: \ell_{u_{2}}^{2^{j d}} \rightarrow m_{u_{2}, p_{2}}^{2^{j d}}\right\| \\
& \sim 2^{-k 2^{-j d}} 2^{j d\left(\frac{1}{u_{2}}-\frac{1}{u_{1}}\right)}
\end{aligned}
$$


since $\|$ id $: \ell_{u_{2}}^{2^{j d}} \rightarrow m_{u_{2}, p_{2}}^{2^{j d}} \|=1$, cf. Lemma 3.4. On the other hand, taking $q=p_{2}$ in 3.8, we get

$$
\begin{aligned}
c 2^{-k 2^{-j d}} 2^{j d\left(\frac{1}{p_{2}}-\frac{1}{u_{1}}\right)} & \leq e_{k}\left(\operatorname{id}_{j}: m_{u_{1}, p_{1}}^{2^{j d}} \rightarrow \ell_{p_{2}}^{2^{j d}}\right) \\
& \leq e_{k}\left(\operatorname{id}_{j}: m_{u_{1}, p_{1}}^{2^{j d}} \rightarrow m_{u_{2}, p_{2}}^{2^{j d}}\right)\left\|\mathrm{id}: m_{u_{2}, p_{2}}^{2^{j d}} \rightarrow \ell_{p_{2}}^{2^{j d}}\right\| \\
& \sim 2^{j d\left(\frac{1}{p_{2}}-\frac{1}{u_{2}}\right)} e_{k}\left(\mathrm{id}_{j}: m_{u_{1}, p_{1}}^{2^{j d}} \rightarrow m_{u_{2}, p_{2}}^{2^{j d}}\right),
\end{aligned}
$$

in view of $\|$ id $: m_{u_{2}, p_{2}}^{2^{j d}} \rightarrow \ell_{p_{2}}^{2^{j d}} \|=2^{j d\left(\frac{1}{p_{2}}-\frac{1}{u_{2}}\right)}$, cf. Lemma 3.4. This proves 3.6.

The next result is well-known in the Banach case, cf. [2], [31, p.63] or [4, Prop. II.1.3]. The extension to the quasi-Banach case is standard.

Lemma 3.7. Let $(E,\|\cdot\|)$ be an $N$-dimensional complex quasi-Banach space. Then

$$
e_{k}(\text { id }: E \rightarrow E) \sim\left\{\begin{array}{lll}
1 & \text { if } & k \leq 2 N \\
2^{-\frac{k-1}{2 N}} & \text { if } & k>2 N
\end{array}\right.
$$

This leads to the following operator ideal estimate.

Lemma 3.8. Let $0<p_{i} \leq u_{i}<\infty, i=1,2$, and $j \in \mathbb{N}_{0}$ be given. Assume that $p_{1} \geq p_{2}$, or $p_{1}<p_{2}$ and $\frac{p_{2}}{u_{2}} \leq \frac{p_{1}}{u_{1}}$. Let $\frac{1}{u}=\frac{1}{u_{1}}-\frac{1}{u_{2}}$. Then

$$
L_{r, \infty}^{(e)}\left(\mathrm{id}: m_{u_{1}, p_{1}}^{2^{j d}} \rightarrow m_{u_{2}, p_{2}}^{2^{j d}}\right) \sim 2^{j d\left(\frac{1}{r}-\frac{1}{u}\right)} \quad \text { if } \frac{1}{r}>\max \left(0, \frac{1}{u}\right) .
$$

Proof. If $u_{2}<p_{1}$, then 3.12 follows from Lemma 3.6.

Let $p_{1} \leq u_{2}$ and let $e_{k}=e_{k}\left(\mathrm{id}_{j}: m_{u_{1}, p_{1}}^{2^{j d}} \rightarrow m_{u_{2}, p_{2}}^{2^{j d}}\right)$. Lemma 3.5 implies

$$
\sup _{k \geq c 2^{j d}} k^{\frac{1}{r}} e_{k} \sim 2^{j d\left(\frac{1}{r}-\frac{1}{u}\right)} .
$$

Thus to verify [3.12] it is sufficient to prove the estimates from above for $1 \leq k \leq c 2^{j d}$. If $\log c 2^{j d} \leq k \leq c 2^{j d}$, then by the Schütt estimates (2.15) we get

$$
\begin{aligned}
k^{\frac{1}{r}} e_{k} & \leq k^{\frac{1}{r}} e_{k}\left(\operatorname{id}_{j}: m_{u_{1}, p_{1}}^{2^{j d}} \rightarrow \ell_{u_{2}}^{2^{j d}}\right) \leq k^{\frac{1}{r}} 2^{j d\left(\frac{1}{p_{1}}-\frac{1}{u_{1}}\right)} e_{k}\left(B_{p_{1}}(0,1), \ell_{u_{2}}\right) \\
& \leq k^{\frac{1}{r}} 2^{j d\left(\frac{1}{u_{2}}-\frac{1}{u_{1}}\right)}\left(\frac{k}{2^{j d}}\right)^{\frac{1}{u_{2}}-\frac{1}{p_{1}}}\left(\log \left(1+\frac{2^{j d}}{k}\right)\right)^{\frac{1}{p_{1}}-\frac{1}{u_{2}}} \leq c 2^{j d\left(\frac{1}{r}+\frac{1}{u_{2}}-\frac{1}{u_{1}}\right)} .
\end{aligned}
$$

If $1 \leq k \leq \log c 2^{j d}$, then Lemma 3.7 implies

$$
\begin{aligned}
k^{\frac{1}{r}} e_{k} & \leq k^{\frac{1}{r}} e_{k}\left(\mathrm{id}: m_{u_{1}, p_{1}}^{2^{j d}} \rightarrow m_{u_{1}, p_{1}}^{2^{j d}}\right)\left\|\mathrm{id}: m_{u_{1}, p_{1}}^{2^{j d}} \rightarrow m_{u_{2}, p_{2}}^{2^{j d}}\right\| \leq k^{\frac{1}{r}} \max \left(1,2^{-j d \frac{1}{u}}\right) \\
& \leq c 2^{j d\left(\frac{1}{r}-\frac{1}{u}\right)},
\end{aligned}
$$

since $k^{1 / r} \leq c 2^{j d\left(\frac{1}{r}-\frac{1}{u}\right)}$ if $\frac{1}{r}>\frac{1}{u}>0$ and $1<k \leq \log c 2^{j d}$. This proves [3.12).

Proposition 3.9. Let $\sigma_{i} \in \mathbb{R}, 0<q_{i} \leq \infty, 0<p_{i} \leq u_{i}<\infty, i=1,2$. Assume $p_{1} \leq u_{2}$ and

$$
\frac{\sigma_{1}-\sigma_{2}}{d}>\frac{1}{p_{1}}-\frac{1}{u_{2}}
$$

Then there is some $c>0$ such that

$$
e_{k}\left(\widetilde{n}_{u_{1}, p_{1}, q_{1}}^{\sigma_{1}} \hookrightarrow \widetilde{n}_{u_{2}, p_{2}, q_{2}}^{\sigma_{2}}\right) \leq c k^{-\frac{\sigma_{1}-\sigma_{2}}{d}} .
$$

Proof. Let $M \in \mathbb{N}$. We decompose the operator id : $\widetilde{n}_{u_{1}, p_{1}, q_{1}}^{\sigma_{1}} \rightarrow \widetilde{n}_{u_{2}, p_{2}, q_{2}}^{\sigma_{2}}$ into the sum of two operators

$$
P_{M}=\sum_{j=0}^{M} \operatorname{id}_{j}, \quad Q_{M}=\sum_{j=0}^{M} \mathrm{id}_{j}, \quad \text { where } \quad\left(\operatorname{id}_{j} \lambda\right)_{\nu, \ell}:= \begin{cases}\lambda_{\nu, \ell} & \text { if } \nu=j, \\ 0 & \text { if } \nu \neq j .\end{cases}
$$


Then by 3.3 we have

$$
\begin{aligned}
e_{k}\left(\operatorname{id}_{j}: \widetilde{n}_{u_{1}, p_{1}, q_{1}}^{\sigma_{1}} \rightarrow \widetilde{n}_{u_{2}, p_{2}, q_{2}}^{\sigma_{2}}\right) & \leq 2^{-j \delta} e_{k}\left(m_{u_{1}, p_{1}}^{2^{j d}} \hookrightarrow m_{u_{2}, p_{2}}^{2^{j d}}\right) \\
& \leq 2^{-j \delta}\left\|m_{u_{1}, p_{1}}^{2^{j d}} \hookrightarrow \ell_{p_{1}}^{2^{j d}}\right\| e_{k}\left(\ell_{p_{1}}^{2^{j d}} \rightarrow \ell_{u_{2}}^{2^{j d}}\right)\left\|\ell_{u_{2}}^{2^{j d}} \hookrightarrow m_{u_{2}, p_{2}}^{2^{j d}}\right\| \\
& \leq C 2^{-j \delta} 2^{j d\left(\frac{1}{p_{1}}-\frac{1}{u_{1}}\right)} e_{k}\left(\ell_{p_{1}}^{2^{j d}} \rightarrow \ell_{u_{2}}^{2^{j d}}\right),
\end{aligned}
$$

where $\delta=\sigma_{1}-\sigma_{2}-\frac{d}{u_{1}}+\frac{d}{u_{2}}$. In consequence,

$$
\begin{aligned}
L_{r, \infty}^{(e)}\left(\mathrm{id}_{j}\right) & \leq C 2^{-j \delta} 2^{j d\left(\frac{1}{p_{1}}-\frac{1}{u_{1}}\right)} 2^{j d\left(\frac{1}{r}+\frac{1}{u_{2}}-\frac{1}{p_{1}}\right)} \\
& =C 2^{j d\left(\frac{1}{r}-\frac{\sigma_{1}-\sigma_{2}}{d}\right)}, \quad \text { if } \frac{1}{r}>\max \left(0, \frac{1}{p_{1}}-\frac{1}{u_{2}}\right),
\end{aligned}
$$

cf. 2.17). We recall, that for any $r>0$ there exist positive numbers $\rho \leq 1$ and $C>0$ such that

$$
L_{r, \infty}^{(e)}\left(\sum_{j} T_{j}\right)^{\varrho} \leq C \sum_{j} L_{r, \infty}^{(e)}\left(T_{j}\right)^{\varrho},
$$

cf. Pietsch [29, 6.2] and König, [18, 1.c.5]. Hence, 3.17) and (3.18) yield

$$
L_{r, \infty}^{(e)}\left(P_{M}\right)^{\varrho} \leq \sum_{j=0}^{M} L_{r, \infty}^{(e)}\left(\mathrm{id}_{j}\right)^{\varrho} \leq c \sum_{j=0}^{M} 2^{\varrho j d\left(\frac{1}{r}-\frac{\sigma_{1}-\sigma_{2}}{d}\right)} \leq c^{\prime} 2^{\varrho M d\left(\frac{1}{r}-\frac{\sigma_{1}-\sigma_{2}}{d}\right)},
$$

if we choose $r$ such that $\frac{1}{r}-\frac{\sigma_{1}-\sigma_{2}}{d}>0$ and $\frac{1}{r}>\frac{1}{p_{1}}-\frac{1}{u_{2}}$. Thus

$$
e_{2^{M d}}\left(P_{M}\right) \leq C 2^{-M d\left(\frac{\sigma_{1}-\sigma_{2}}{d}\right)} .
$$

Similarly,

$$
L_{r, \infty}^{(e)}\left(Q_{M}\right)^{\varrho} \leq \sum_{j=M+1}^{\infty} L_{r, \infty}^{(e)}\left(\mathrm{id}_{j}\right)^{\varrho} \leq c \sum_{j=M+1}^{\infty} 2^{\varrho j d\left(\frac{1}{r}-\frac{\sigma_{1}-\sigma_{2}}{d}\right)} \leq c^{\prime} 2^{\varrho M d\left(\frac{1}{r}-\frac{\sigma_{1}-\sigma_{2}}{d}\right)},
$$

if we choose $r$ such that $0 \leq \frac{1}{p_{1}}-\frac{1}{u_{2}}<\frac{1}{r}<\frac{\sigma_{1}-\sigma_{2}}{d}$. Thus

$$
e_{2^{M d}}\left(Q_{M}\right) \leq C 2^{-M d\left(\frac{\sigma_{1}-\sigma_{2}}{d}\right)} .
$$

Now by (3.19) and 3.20 we can conclude that

$$
e_{2^{M d}}\left(\mathrm{id}: \widetilde{n}_{u_{1}, p_{1}, q_{1}}^{\sigma_{1}} \rightarrow \widetilde{n}_{u_{2}, p_{2}, q_{2}}^{\sigma_{2}}\right) \leq C 2^{-M d\left(\frac{\sigma_{1}-\sigma_{2}}{d}\right)}
$$

and by standard arguments $e_{k}\left(\mathrm{id}: \widetilde{n}_{u_{1}, p_{1}, q_{1}}^{\sigma_{1}} \rightarrow \widetilde{n}_{u_{2}, p_{2}, q_{2}}^{\sigma_{2}}\right) \leq C k^{-\frac{\sigma_{1}-\sigma_{2}}{d}}, k \in \mathbb{N}$.

Now we are ready to establish our first main result in this context which reflects the 'classical' situation as we shall see below.

Theorem 3.10. Let $\sigma_{i} \in \mathbb{R}, 0<q_{i} \leq \infty, 0<p_{i} \leq u_{i}<\infty, i=1,2$. Assume that

(i) either

$$
\frac{\sigma_{1}-\sigma_{2}}{d}>\max \left\{0, \frac{1}{u_{1}}-\frac{1}{u_{2}}, \frac{p_{1}}{u_{1}}\left(\frac{1}{p_{1}}-\frac{1}{p_{2}}\right)\right\}=0,
$$

(ii) or

$$
\frac{\sigma_{1}-\sigma_{2}}{d}>\max \left\{0, \frac{1}{u_{1}}-\frac{1}{u_{2}}, \frac{p_{1}}{u_{1}}\left(\frac{1}{p_{1}}-\frac{1}{p_{2}}\right)\right\}=\frac{1}{u_{1}}-\frac{1}{u_{2}}>0 .
$$

Then

$$
e_{k}\left(\widetilde{n}_{u_{1}, p_{1}, q_{1}}^{\sigma_{1}} \hookrightarrow \widetilde{n}_{u_{2}, p_{2}, q_{2}}^{\sigma_{2}}\right) \sim k^{-\frac{\sigma_{1}-\sigma_{2}}{d}} .
$$


Proof. Step 1. We prove (i). It follows from (3.22) that $u_{2} \leq u_{1}$ and $p_{2} \leq p_{1}$. So we may consider two cases: $p_{2} \leq u_{2}<p_{1} \leq u_{2}$ or $p_{2} \leq p_{1} \leq u_{2} \leq u_{1}$.

Substep 1.1. In the first case Lemma 3.6 implies that

$$
L_{r, \infty}^{(e)}\left(\mathrm{id}: m_{u_{1}, p_{1}}^{2^{j d}} \hookrightarrow m_{u_{2}, p_{2}}^{2^{j d}}\right) \sim 2^{j d\left(\frac{1}{r}-\frac{1}{u_{1}}+\frac{1}{u_{2}}\right)}, \quad \frac{1}{r}>0 .
$$

In consequence, $L_{r, \infty}^{(e)}\left(\operatorname{id}_{j}\right) \sim 2^{j d\left(\frac{1}{r}-\frac{\sigma_{1}-\sigma_{2}}{d}\right)}$ for any $\frac{1}{r}>0$. Now we can prove the estimate from above in the same way as in Proposition 3.9. The estimate from below follows from Lemma 3.6 and the obvious inequality

$$
e_{2^{j d}}\left(m_{u_{1}, p_{1}}^{2^{j d}} \hookrightarrow m_{u_{2}, p_{2}}^{2^{j d}}\right) \leq 2^{j \delta} e_{2^{j d}}\left(\widetilde{n}_{u_{1}, p_{1}, q_{1}}^{\sigma_{1}} \hookrightarrow \widetilde{n}_{u_{2}, p_{2}, q_{2}}^{\sigma_{2}}\right) .
$$

Substep 1.2. Let now $p_{2} \leq p_{1} \leq u_{2} \leq u_{1}$. We consider the diagram

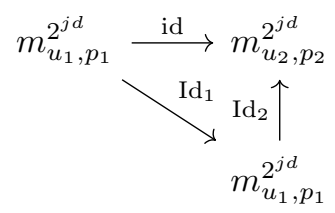

By Lemma 3.4 we have $\left\|\operatorname{id}_{2}\right\|=2^{j d\left(\frac{1}{u_{2}}-\frac{1}{u_{1}}\right)}$. Moreover Lemma 3.7 implies $L_{r, \infty}^{(e)}\left(\operatorname{Id}_{1}\right) \leq c 2^{\frac{j d}{r}}$. In consequence,

$$
L_{r, \infty}^{(e)}\left(\operatorname{id}_{j}\right) \leq 2^{-j \delta} 2^{j d\left(\frac{1}{u_{2}}-\frac{1}{u_{1}}\right)} L_{r, \infty}^{(e)}\left(\operatorname{Id}_{1}\right) \leq 2^{j d\left(\frac{1}{r}-\frac{\sigma_{1}-\sigma_{2}}{d}\right)} .
$$

In the same way as above we show that $e_{2^{M d}}\left(P_{M}\right) \leq C 2^{-M d\left(\frac{\sigma_{1}-\sigma_{2}}{d}\right)}$ and $e_{2^{M d}}\left(Q_{M}\right) \leq$ $C 2^{-M d\left(\frac{\sigma_{1}-\sigma_{2}}{d}\right)}$. The estimate from below follows from Lemma 3.5 ,

Step 2. We prove (ii). Let $p_{1} \geq p_{2}$ and $u_{1}<u_{2}$, or $p_{1}<p_{2}$ and $\frac{p_{2}}{u_{2}} \leq \frac{p_{1}}{u_{1}}$. In both cases we can use Lemma 3.8. So the estimate from above can be proved in the same way as in Proposition 3.9 . The estimate from below follows once more from Lemma 3.5.

Remark 3.11. We call this the 'classical' setting, as in case of $p=u$ the sequence spaces $\tilde{n}_{p, p, q}^{\sigma}=\tilde{b}_{p, q}^{\sigma}$ coincide, recall Remark 3.2. As mentioned in 3.1 , it is well-known that $e_{k}\left(\tilde{b}_{u_{1}, q_{1}}^{\sigma_{1}} \hookrightarrow\right.$ $\left.\tilde{b}_{p_{2}, q_{2}}^{\sigma_{2}}\right) \sim k^{-\frac{\sigma_{1}-\sigma_{2}}{d}}$ in all cases admitted by (2.10); see [45, Thm. 8.2]. This behaviour is now extended to spaces $\widetilde{n}_{u, p, q}^{\sigma}$ for all $0<p \leq u<\infty$ whenever 3.22) or 3.23) are satisfied.

In view of our compactness result Theorem 2.10 , in particular, 2.10 , and Theorem 3.10 , it remains to deal with the case when

$$
\max \left\{0, \frac{1}{u_{1}}-\frac{1}{u_{2}}\right\}<\frac{p_{1}}{u_{1}}\left(\frac{1}{p_{1}}-\frac{1}{p_{2}}\right)
$$

which is equivalent to $p_{1}<p_{2}$ and $\frac{p_{2}}{u_{2}}>\frac{p_{1}}{u_{1}}$. This excludes, in particular, the setting $p_{1}=u_{1}$. We first give the counterpart of Proposition 3.9 in this case.

Proposition 3.12. Let $\sigma_{i} \in \mathbb{R}, 0<q_{i} \leq \infty, 0<p_{i} \leq u_{i}<\infty, i=1,2$. Assume that $p_{1}<p_{2}, \frac{p_{1}}{u_{1}}<\frac{p_{2}}{u_{2}}$ and

$$
\frac{\sigma_{1}-\sigma_{2}}{d}>\frac{1}{p_{1}}-\frac{1}{p_{2}}
$$

Then there is some $c>0$ such that

$$
e_{k}\left(\widetilde{n}_{u_{1}, p_{1}, q_{1}}^{\sigma_{1}} \hookrightarrow \widetilde{n}_{u_{2}, p_{2}, q_{2}}^{\sigma_{2}}\right) \leq c k^{-\frac{\sigma_{1}-\sigma_{2}}{d}} .
$$

Proof. Step 1. First we prove that

$$
L_{r, \infty}^{(e)}\left(m_{u_{1}, p_{1}}^{2^{j d}} \hookrightarrow m_{u_{2}, p_{2}}^{2^{j d}}\right) \leq c 2^{j d\left(\frac{1}{r}-\frac{1}{u_{1}}+\frac{1}{u_{2}}\right)}, \quad \text { whenever } \frac{1}{r}>\frac{1}{p_{1}}-\frac{1}{p_{2}} .
$$

Since $p_{1}<p_{2}$ we have for any $\nu, 0 \leq \nu \leq j$,

$$
\begin{aligned}
2^{(j-\nu) d\left(\frac{p_{2}}{u_{2}}-1\right)} & \sum_{k: Q_{j, k} \subset Q_{\nu, m}}\left|\lambda_{j, k}\right|^{p_{2}} \\
\leq & 2^{(j-\nu) d\left(\frac{p_{2}}{u_{2}}-\frac{p_{1}}{u_{1}}\right)}\left(\sup _{k: Q_{j, k} \subset Q_{\nu, m}}\left|\lambda_{j, k}\right|\right)^{p_{2}-p_{1}} 2^{(j-\nu) d\left(\frac{p_{1}}{u_{1}}-1\right)} \sum_{k: Q_{j, k} \subset Q_{\nu, m}}\left|\lambda_{j, k}\right|^{p_{1}} .
\end{aligned}
$$


Moreover $\frac{p_{2}}{u_{2}}>\frac{p_{1}}{u_{1}}$, so taking the supremum we get

$$
\left\|\left.\lambda\left|m_{u_{2}, p_{2}}^{2^{j d}}\left\|\leq 2^{j d\left(\frac{1}{u_{2}}-\frac{p_{1}}{p_{2} u_{1}}\right)}\right\| \lambda\right|\right|_{\infty} ^{2^{j d}}\right\|^{1-\frac{p_{1}}{p_{2}}}\left\|\lambda \mid m_{u_{1}, p_{1}}^{2^{j d}}\right\|^{\frac{p_{1}}{p_{2}}} .
$$

Let $m_{u_{2}, p_{2}}^{*}$ denote the space $m_{u_{2}, p_{2}}^{2^{j d}}$ equipped with the norm

$$
\left\|\lambda\left|m_{u_{2}, p_{2}}^{*}\left\|=2^{-j d\left(\frac{1}{u_{2}}-\frac{p_{1}}{p_{2} u_{1}}\right)}\right\| \lambda\right| m_{u_{2}, p_{2}}^{2^{j d}}\right\| .
$$

Thus 3.31 implies

$$
\left\|\lambda\left|m_{u_{2}, p_{2}}^{*}\|\leq\| \lambda\right| \ell_{\infty}^{2^{j d}}\right\|^{1-\frac{p_{1}}{p_{2}}}\left\|\lambda \mid m_{u_{1}, p_{1}}^{2^{j d}}\right\|^{\frac{p_{1}}{p_{2}}} .
$$

Now we estimate the entropy numbers of the embedding $m_{u_{1}, p_{1}}^{2^{j d}} \hookrightarrow m_{u_{2}, p_{2}}^{*}$. Lemma 3.4 implies

$$
e_{k}\left(m_{u_{1}, p_{1}}^{2^{j d}} \hookrightarrow \ell_{\infty}^{2^{j d}}\right) \leq 2^{j d\left(\frac{1}{p_{1}}-\frac{1}{u_{1}}\right)} e_{k}\left(\ell_{p_{1}}^{2^{j d}} \hookrightarrow \ell_{\infty}^{2^{j d}}\right)
$$

So using the inequality 3.33 , Schütt's estimates (2.15), Lemma 3.7 and the interpolation properties of the entropy numbers $(2.12,2.13)$ with $\theta=\frac{p_{1}}{p_{2}}$, we get

$$
\begin{aligned}
& e_{2 k-1}\left(m_{u_{1}, p_{1}}^{2^{j d}} \hookrightarrow m_{u_{2}, p_{2}}^{*}\right) \\
& \quad \leq c e_{k}\left(m_{u_{1}, p_{1}}^{2^{j d}} \hookrightarrow \ell_{\infty}^{2^{j d}}\right)^{1-\frac{p_{1}}{p_{2}}} e_{k}\left(m_{u_{1}, p_{1}}^{2^{j d}} \hookrightarrow m_{u_{1}, p_{1}}^{2^{j d}}\right)^{\frac{p_{1}}{p_{2}}} \\
& \quad \leq c 2^{j d\left(\frac{1}{p_{1}}-\frac{1}{u_{1}}\right)\left(1-\frac{p_{1}}{p_{2}}\right)} \begin{cases}1 & \text { if } 1 \leq k \leq \log 2^{j d+1}, \\
\left(k^{-1} \log \left(\left(1+\frac{2^{j d}}{k}\right)\right)\right)^{\frac{1}{p_{1}}-\frac{1}{p_{2}}} & \text { if } \log 2^{j d+1} \leq k \leq 2^{j d+1}, \\
2^{-k 2^{-j d}} 2^{-j d\left(\frac{1}{p_{1}}-\frac{1}{p_{2}}\right)} & \text { if } k \geq 2^{j d+1} .\end{cases}
\end{aligned}
$$

Let us take $r>0$ such that $\frac{1}{r}>\frac{1}{p_{1}}-\frac{1}{p_{2}}$. Then using the estimates (3.35) we can prove that

$$
k^{\frac{1}{r}} e_{k}\left(m_{u_{1}, p_{1}}^{2^{j d}} \hookrightarrow m_{u_{2}, p_{2}}^{*}\right) \leq c 2^{j d\left(\frac{1}{r}-\frac{1}{p_{1}}+\frac{1}{p_{2}}\right)} 2^{j d\left(\frac{1}{p_{1}}-\frac{1}{u_{1}}\right)\left(1-\frac{p_{1}}{p_{2}}\right)}=2^{j d\left(\frac{1}{r}-\frac{p_{1}}{u_{1}}\left(\frac{1}{p_{1}}-\frac{1}{p_{2}}\right)\right)} .
$$

But the relation 3.32 between the norms leads to

$$
e_{k}\left(m_{u_{1}, p_{1}}^{2^{j d}} \hookrightarrow m_{u_{2}, p_{2}}^{2^{j d}}\right)=2^{j d\left(\frac{1}{u_{2}}-\frac{p_{1}}{p_{2} u_{1}}\right)} e_{k}\left(m_{u_{1}, p_{1}}^{2^{j d}} \hookrightarrow m_{u_{2}, p_{2}}^{*}\right)
$$

So 3.36 gives

$$
k^{\frac{1}{r}} e_{k}\left(m_{u_{1}, p_{1}}^{2^{j d}} \hookrightarrow m_{u_{2}, p_{2}}^{2^{j d}}\right) \leq c 2^{j d\left(\frac{1}{r}-\frac{1}{u_{1}}+\frac{1}{u_{2}}\right)} .
$$

This proves 3.30 .

Step 2. We proceed similar to the proof of Proposition 3.9, Let $M \in \mathbb{N}$. We decompose the operator id : $\widetilde{n}_{u_{1}, p_{1}, q_{1}}^{\sigma_{1}} \rightarrow \widetilde{n}_{u_{2}, p_{2}, q_{2}}^{\sigma_{2}}$ as in (3.16).

The elementary properties of entropy numbers imply

$$
e_{k}\left(\operatorname{id}_{j}: \widetilde{n}_{u_{1}, p_{1}, q_{1}}^{\sigma_{1}} \rightarrow \widetilde{n}_{u_{2}, p_{2}, q_{2}}^{\sigma_{2}}\right) \leq 2^{-j \delta} e_{k}\left(m_{u_{1}, p_{1}}^{2^{j d}} \hookrightarrow m_{u_{2}, p_{2}}^{2^{j d}}\right)
$$

where $\delta=\sigma_{1}-\sigma_{2}-\frac{d}{u_{1}}+\frac{d}{u_{2}}$. Consequently, using (3.30), 3.37) and (3.38) we arrive at

$$
L_{r, \infty}^{(e)}\left(\operatorname{id}_{j}\right) \leq C 2^{-j \delta} 2^{j d\left(\frac{1}{r}-\frac{1}{u_{1}}+\frac{1}{u_{2}}\right)}=C 2^{j d\left(\frac{1}{r}-\frac{\sigma_{1}-\sigma_{2}}{d}\right)}, \quad \frac{1}{r}>\frac{1}{p_{1}}-\frac{1}{p_{2}} .
$$

Hence for some positive number $\rho \leq 1$, 3.39 and 3.18 yield

$$
L_{r, \infty}^{(e)}\left(P_{M}\right)^{\varrho} \leq \sum_{j=0}^{M} L_{r, \infty}^{(e)}\left(\mathrm{id}_{j}\right)^{\varrho} \leq c \sum_{j=0}^{M} 2^{\varrho j d\left(\frac{1}{r}-\frac{\sigma_{1}-\sigma_{2}}{d}\right)} \leq c^{\prime} 2^{\varrho M d\left(\frac{1}{r}-\frac{\sigma_{1}-\sigma_{2}}{d}\right)},
$$

if we choose $r$ such that $\frac{1}{r}>\frac{\sigma_{1}-\sigma_{2}}{d}>\frac{1}{p_{1}}-\frac{1}{p_{2}}$. Thus

$$
e_{2^{M d}}\left(P_{M}\right) \leq C 2^{-M d\left(\frac{\sigma_{1}-\sigma_{2}}{d}\right)} .
$$


Similarly,

$$
L_{r, \infty}^{(e)}\left(Q_{M}\right)^{\varrho} \leq \sum_{j=M+1}^{\infty} L_{r, \infty}^{(e)}\left(\mathrm{id}_{j}\right)^{\varrho} \leq c \sum_{j=M+1}^{\infty} 2^{\varrho j d\left(\frac{1}{r}-\frac{\sigma_{1}-\sigma_{2}}{d}\right)} \leq c^{\prime} 2^{\varrho M d\left(\frac{1}{r}-\frac{\sigma_{1}-\sigma_{2}}{d}\right)},
$$

if we choose $r$ this time such that $0 \leq \frac{1}{p_{1}}-\frac{1}{p_{2}}<\frac{1}{r}<\frac{\sigma_{1}-\sigma_{2}}{d}$. Thus

$$
e_{2^{M d}}\left(Q_{M}\right) \leq C 2^{-M d\left(\frac{\sigma_{1}-\sigma_{2}}{d}\right)} .
$$

Now 3.40 and 3.41 give us $e_{2^{M d}}\left(\mathrm{id}: \widetilde{n}_{u_{1}, p_{1}, q_{1}}^{\sigma_{1}} \rightarrow \widetilde{n}_{u_{2}, p_{2}, q_{2}}^{\sigma_{2}}\right) \leq C 2^{-M d\left(\frac{\sigma_{1}-\sigma_{2}}{d}\right)}$ and by standard arguments $e_{k}\left(\mathrm{id}: \widetilde{n}_{u_{1}, p_{1}, q_{1}}^{\sigma_{1}} \rightarrow \widetilde{n}_{u_{2}, p_{2}, q_{2}}^{\sigma_{2}}\right) \leq C k^{-\frac{\sigma_{1}-\sigma_{2}}{d}}, k \in \mathbb{N}$, as desired.

Now we can present the (almost) complete counterpart of Theorem 3.10 showing some surprising phenomenon.

Theorem 3.13. Let $\sigma_{i} \in \mathbb{R}, 0<q_{i} \leq \infty, 0<p_{i} \leq u_{i}<\infty, i=1,2$, and

$$
\frac{\sigma_{1}-\sigma_{2}}{d}>\frac{p_{1}}{u_{1}}\left(\frac{1}{p_{1}}-\frac{1}{p_{2}}\right)>\max \left\{0, \frac{1}{u_{1}}-\frac{1}{u_{2}}\right\} .
$$

(i) If $\frac{\sigma_{1}-\sigma_{2}}{d}>\frac{1}{p_{1}}-\frac{1}{p_{2}}$, then

$$
e_{k}\left(\widetilde{n}_{u_{1}, p_{1}, q_{1}}^{\sigma_{1}} \hookrightarrow \widetilde{n}_{u_{2}, p_{2}, q_{2}}^{\sigma_{2}}\right) \sim k^{-\frac{\sigma_{1}-\sigma_{2}}{d}} .
$$

(ii) If $\frac{p_{1}}{u_{1}}\left(\frac{1}{p_{1}}-\frac{1}{p_{2}}\right)<\frac{\sigma_{1}-\sigma_{2}}{d} \leq \frac{1}{p_{1}}-\frac{1}{p_{2}}$, then there exists some $c>0$ and for any $\varepsilon>0$ some $c_{\varepsilon}>0$ such that for all $k \in \mathbb{N}$,

$$
\begin{aligned}
c k^{-\frac{u_{1}}{u_{1}-p_{1}}\left(\frac{\sigma_{1}-\sigma_{2}}{d}-\frac{p_{1}}{u_{1}}\left(\frac{1}{p_{1}}-\frac{1}{p_{2}}\right)\right)} & \leq e_{k}\left(\widetilde{n}_{u_{1}, p_{1}, q_{1}}^{\sigma_{1}} \hookrightarrow \widetilde{n}_{u_{2}, p_{2}, q_{2}}^{\sigma_{2}}\right) \\
& \leq c_{\varepsilon} k^{-\frac{u_{1}}{u_{1}-p_{1}}\left(\frac{\sigma_{1}-\sigma_{2}}{d}-\frac{p_{1}}{u_{1}}\left(\frac{1}{p_{1}}-\frac{1}{p_{2}}\right)\right)+\varepsilon} .
\end{aligned}
$$

Proof. Recall that the condition 3.42 , that is,

$$
\max \left\{0, \frac{1}{u_{1}}-\frac{1}{u_{2}}\right\}<\frac{p_{1}}{u_{1}}\left(\frac{1}{p_{1}}-\frac{1}{p_{2}}\right)
$$

is equivalent to $p_{1}<p_{2}$ and $\frac{p_{2}}{u_{2}}>\frac{p_{1}}{u_{1}}$. All other cases are already covered by Theorem 3.10 ,

Step 1. We prove (i). The statement follows immediately from Proposition 3.12 and Lemma 3.5 ,

Step 2. We first show the upper estimate in (ii). Here we make use of the interpolation result, Theorem 2.12 , in its sequence space version, together with the interpolation property of entropy numbers [2.13).

Let $\varepsilon_{1}>0$ and $\tau_{1}=\sigma_{2}+d\left(\frac{1}{p_{1}}-\frac{1}{p_{2}}\right)+d \varepsilon_{1}$ be such that $\tau_{1}-\sigma_{2}>d\left(\frac{1}{p_{1}}-\frac{1}{p_{2}}\right)$. Hence we can apply (i) to the compact embedding $\operatorname{id}_{1}: \widetilde{n}_{u_{1}, p_{1}, v_{1}}^{\tau_{1}} \hookrightarrow \widetilde{n}_{u_{2}, p_{2}, q_{2}}^{\sigma_{2}}$, where $0<v_{1} \leq \infty$ is arbitrary. Thus (3.43) leads to

$$
e_{k}\left(\operatorname{id}_{1}: \widetilde{n}_{u_{1}, p_{1}, v_{1}}^{\tau_{1}} \hookrightarrow \widetilde{n}_{u_{2}, p_{2}, q_{2}}^{\sigma_{2}}\right) \leq c k^{-\frac{\tau_{1}-\sigma_{2}}{d}}=c_{\varepsilon_{1}} k^{-\left(\frac{1}{p_{1}}-\frac{1}{p_{2}}\right)-\varepsilon_{1}} .
$$

We choose $\tau_{2}=\sigma_{2}+d \frac{p_{1}}{u_{1}}\left(\frac{1}{p_{1}}-\frac{1}{p_{2}}\right)$ and $v_{2}$ with $0<v_{2} \leq \min \left(1, \max \left(1, \frac{u_{2}}{u_{1}}\right) \frac{p_{1}}{p_{2}}\right) q_{2}$. Then by [13, Thm. 3.1] the embedding $\operatorname{id}_{2}: \widetilde{n}_{u_{1}, p_{1}, v_{2}}^{\tau_{2}} \hookrightarrow \widetilde{n}_{u_{2}, p_{2}, q_{2}}^{\sigma_{2}}$ is continuous, that is,

$e_{k}\left(\mathrm{id}_{2}: \widetilde{n}_{u_{1}, p_{1}, v_{2}}^{\tau_{2}} \hookrightarrow \widetilde{n}_{u_{2}, p_{2}, q_{2}}^{\sigma_{2}}\right) \leq c$. Now we determine the number $\theta \in(0,1)$ appropriately such that $\sigma_{1}=(1-\theta) \tau_{1}+\theta \tau_{2}$, which is always possible in case (ii). The sequence space version of Theorem 2.12 i) yields then

$$
\widetilde{n}_{u_{1}, p_{1}, q_{1}}^{\sigma_{1}}=\left(\widetilde{n}_{u_{1}, p_{1}, v_{1}}^{\tau_{1}}, \widetilde{n}_{u_{1}, p_{1}, v_{2}}^{\tau_{2}}\right)_{\theta, q_{1}} .
$$

Thus the interpolation property 2.13 leads to

$$
e_{2 k}\left(\widetilde{n}_{u_{1}, p_{1}, q_{1}}^{\sigma_{1}} \hookrightarrow \widetilde{n}_{u_{2}, p_{2}, q_{2}}^{\sigma_{2}}\right) \leq c e_{k}\left(\mathrm{id}_{1}\right)^{1-\theta} e_{k}\left(\mathrm{id}_{2}\right)^{\theta} \leq c_{\varepsilon_{1}}^{\prime} k^{-(1-\theta)\left(\frac{1}{p_{1}}-\frac{1}{p_{2}}+\varepsilon_{1}\right)}
$$


and it remains to show that

$$
-\frac{u_{1}}{u_{1}-p_{1}}\left(\frac{\sigma_{1}-\sigma_{2}}{d}-\frac{p_{1}}{u_{1}}\left(\frac{1}{p_{1}}-\frac{1}{p_{2}}\right)\right)+\varepsilon=-(1-\theta)\left(\frac{1}{p_{1}}-\frac{1}{p_{2}}+\varepsilon_{1}\right)
$$

and appropriately chosen $\varepsilon$ (in dependence on $\varepsilon_{1}$ ). Moreover, $\varepsilon_{1} \rightarrow 0$ implies $\varepsilon \rightarrow 0$. However, this is obvious by straightforward calculation.

Step 3. It remains to show the lower estimate in (ii). Let $k_{j}=\left\lfloor 2^{j d\left(1-\frac{p_{1}}{u_{1}}\right)}\right\rfloor$. We consider the following commutative diagram

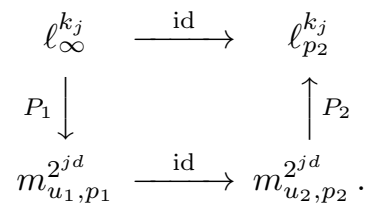

The operator $P_{1}$ is defined in the following way. Let $\lambda^{(j)}=\left(\lambda_{\ell}^{(j)}\right)$ be a sequence constructed in Substep 2.2 of the proof of Theorem 2.1 in [15]. The sequence has $k_{j}$ elements equal to 1 and the remaining elements are 0 . Moreover $\left\|\lambda^{(j)} \mid m_{u_{1}, p_{1}}^{2^{j d}}\right\|=1$. Let $T$ be a one-to-one correspondence between the set $\left\{1, \ldots, k_{j}\right\}$ and the cubes $Q_{0, \ell}$ such that $\lambda_{\ell}^{(j)}=1$. For $\mu=$ $\left(\mu_{m}\right) \in \ell_{\infty}^{k_{j}}$ we put

$$
P_{1}(\mu)_{\ell}= \begin{cases}\mu_{m} & \text { if } \quad T(m)=Q_{0, \ell} \\ 0 & \text { otherwise. }\end{cases}
$$

Then $P_{1}$ is a linear operator mapping $\ell_{\infty}^{k_{j}}$ into $m_{u_{1}, p_{1}}^{2^{j d}}$ and it norm equals 1 .

The operator $P_{2}$ is a projection, $P_{2}(\lambda)_{m}=\lambda_{\ell}$ if $T(m)=Q_{0, \ell}$. Then Lemma 3.4 implies that $\left\|P_{2}\right\| \leq 2^{j d\left(\frac{1}{p_{2}}-\frac{1}{u_{2}}\right)}$. We conclude from the above diagram and Schütt's estimates that

$$
c 2^{j d\left(\frac{1}{u_{2}}-\frac{p_{1}}{u_{1} p_{2}}\right)} \leq e_{k_{j}}\left(m_{u_{1}, p_{1}}^{2^{j d}} \hookrightarrow m_{u_{2}, p_{2}}^{2^{j d}}\right) .
$$

This finally leads to

$$
\begin{aligned}
c k_{j}^{-\frac{u_{1}}{u_{1}-p_{1}}\left(\frac{\sigma_{1}-\sigma_{2}}{d}-\frac{p_{1}}{u_{1}}\left(\frac{1}{p_{1}}-\frac{1}{p_{2}}\right)\right)} & =c 2^{-j d\left(\frac{\sigma_{1}-\sigma_{2}}{d}-\frac{p_{1}}{u_{1}}\left(\frac{1}{p_{1}}-\frac{1}{p_{2}}\right)\right)} \\
& \leq e_{k_{j}}\left(\widetilde{n}_{u_{1}, p_{1}, q_{1}}^{\sigma_{1}} \hookrightarrow \widetilde{n}_{u_{2}, p_{2}, q_{2}}^{\sigma_{2}}\right) .
\end{aligned}
$$

Remark 3.14. Note that the 'classical' exponent $\frac{\sigma_{1}-\sigma_{2}}{d}$ of the asymptotic decay of entropy numbers, as it appears in Theorem 3.10 already, is extended in part (i) of the above theorem for $\sigma_{1}-\sigma_{2}$ 'large enough', that is, $\sigma_{1}-\sigma_{2}>d\left(\frac{1}{p_{1}}-\frac{1}{p_{2}}\right)$. However, for small differences $\sigma_{1}-\sigma_{2}$ and in the proper Morrey case for the source space as dealt with in (ii), which does not exist when $p_{1}=u_{1}$, we can at the first time observe a completely different behaviour for the asymptotic decay of the entropy numbers. In particular, if $\frac{p_{1}}{u_{1}}\left(\frac{1}{p_{1}}-\frac{1}{p_{2}}\right)<\frac{\sigma_{1}-\sigma_{2}}{d}<\frac{1}{p_{1}}-\frac{1}{p_{2}}$, then $\frac{u_{1}}{u_{1}-p_{1}}\left(\frac{\sigma_{1}-\sigma_{2}}{d}-\frac{p_{1}}{u_{1}}\left(\frac{1}{p_{1}}-\frac{1}{p_{2}}\right)\right)<\frac{\sigma_{1}-\sigma_{2}}{d}$. So in that case the power $\frac{\sigma_{1}-\sigma_{2}}{d}$ does not describe the asymptotic behaviour of entropy numbers, and their decay is much slower than usual.

On the other hand, if $\sigma_{1}-\sigma_{2}=d\left(\frac{1}{p_{1}}-\frac{1}{p_{2}}\right)$, that is, when the above cases (i) and (ii) meet, then the upper estimate in (3.44) reads as

$$
e_{k}\left(\widetilde{n}_{u_{1}, p_{1}, q_{1}}^{\sigma_{1}} \hookrightarrow \widetilde{n}_{u_{2}, p_{2}, q_{2}}^{\sigma_{2}}\right) \leq c_{\varepsilon} k^{-\frac{\sigma_{1}-\sigma_{2}}{d}+\varepsilon},
$$

that is, the same exponent (up to $\varepsilon$ ) as in (3.43). This gives some hint that the exponent in (3.44) might be close to the precise description. We postpone some further discussion to Remark 4.5 below.

\section{Entropy numbers of compact embeddings in smoothness Morrey spaces on bounded domains}

First we note that the asymptotic behaviour of the entropy numbers $e_{k}\left(\operatorname{id}_{\mathcal{A}}: \mathcal{A}_{u_{1}, p_{1}, q_{1}}^{s_{1}}(\Omega) \hookrightarrow\right.$ $\left.\mathcal{A}_{u_{2}, p_{2}, q_{2}}^{s_{2}}(\Omega)\right)$ for an arbitrary domain, i.e., an open bounded set $\Omega$ in $\mathbb{R}^{d}$, is the same as for a $C^{\infty}$ bounded domain. So we can concentrate on $C^{\infty}$ bounded domains afterwards. 
Lemma 4.1. Let $\Omega$ be a non-empty open bounded set and $B_{e}(0,1)$ be an open unit ball in $\mathbb{R}^{d}$. Then the embedding $\mathcal{A}_{u_{1}, p_{1}, q_{1}}^{s_{1}}(\Omega) \hookrightarrow \mathcal{A}_{u_{2}, p_{2}, q_{2}}^{s_{2}}(\Omega)$ is compact if and only if the embedding $\mathcal{A}_{u_{1}, p_{1}, q_{1}}^{s_{1}}\left(B_{e}(0,1)\right) \hookrightarrow \mathcal{A}_{u_{2}, p_{2}, q_{2}}^{s_{2}}\left(B_{e}(0,1)\right)$ is compact. Moreover, in that case,

$$
\left.e_{k}\left(\mathcal{A}_{u_{1}, p_{1}, q_{1}}^{s_{1}}(\Omega) \hookrightarrow \mathcal{A}_{u_{2}, p_{2}, q_{2}}^{s_{2}}(\Omega)\right) \sim e_{k}\left(\mathcal{A}_{u_{1}, p_{1}, q_{1}}^{s_{1}}\left(B_{e}(0,1)\right) \hookrightarrow \mathcal{A}_{u_{2}, p_{2}, q_{2}}^{s_{2}}\left(B_{e}(0,1)\right)\right)\right) .
$$

Proof. The lemma holds for any ball $\Omega=B_{e}(x, R)$, centred at $x \in \mathbb{R}^{d}$ with radius $R>0$, since translations and dilations define bounded operators in the spaces $\mathcal{A}_{u, p, q}^{s}\left(\mathbb{R}^{d}\right)$. To prove the statement for general $\Omega$ it is sufficient to prove 4.1 since an embedding is compact if, and only if, its entropy numbers $e_{k}$ tend to zero for $k \rightarrow \infty$.

Let $B_{e}(0, R)$ be an open ball such that $\bar{\Omega} \subset B_{e}(0, R)$. Multiplication by a smooth compactly supported function defines a bounded operator in $\mathcal{A}_{u, p, q}^{s}\left(\mathbb{R}^{d}\right)$, therefore the expression

$$
\left\|f \mid \mathcal{A}_{u, p, q}^{s}(\Omega)\right\|_{*}=\inf \left\{\left\|g\left|\mathcal{A}_{u, p, q}^{s}\left(\mathbb{R}^{d}\right) \|: \quad f=g\right|_{\Omega} \quad \text { and } \quad \operatorname{supp} g \subset B_{e}(0, R)\right\}\right.
$$

is an equivalent norm in $\mathcal{A}_{u, p, q}^{s}(\Omega)$. Let $B_{i}(g, r)$ denote a ball in the space $\mathcal{A}_{u_{i}, p_{i}, q_{i}}^{s_{i}}(\Omega)$ centred at $g$ with radius $r$, and let $\widetilde{B}_{i}(g, r)$ denote a corresponding ball in $\mathcal{A}_{u_{i}, p_{i}, q_{i}}^{s_{i}}\left(B_{e}(0, R)\right), i=1,2$.

Let $c>1$ and $f \in B_{1}(0,1)$. Then there exists some $g \in \mathcal{A}_{u_{1}, p_{1}, q_{1}}^{s_{1}}\left(B_{e}(0, R)\right)$ such that $f=\left.g\right|_{\Omega}$ and $\left\|g\left|\mathcal{A}_{u_{1}, p_{1}, q_{1}}^{s_{1}}\left(B_{e}(x, R)\right)\|\leq c\| f\right| \mathcal{A}_{u_{1}, p_{1}, q_{1}}^{s_{1}}(\Omega)\right\|_{*}<c$. Let $\varepsilon>0$ and choose $k \in \mathbb{N}$ sufficiently large such that

$$
e_{k}\left(\mathcal{A}_{u_{1}, p_{1}, q_{1}}^{s_{1}}\left(B_{e}(x, R)\right) \hookrightarrow \mathcal{A}_{u_{2}, p_{2}, q_{2}}^{s_{2}}\left(B_{e}(x, R)\right)\right)<\varepsilon .
$$

The definition of entropy numbers implies that there are $g_{j} \in \mathcal{A}_{u_{2}, p_{2}, q_{2}}^{s_{2}}\left(B_{e}(x, R)\right), j=1, \ldots, 2^{k-1}$, such that

$$
\widetilde{B}_{1}(0, c) \subset \bigcup_{j=1}^{2^{k-1}} \widetilde{B}_{2}\left(g_{j}, c \varepsilon\right) .
$$

Thus there exists $g_{m}, m \in\left\{1, \ldots, 2^{k-1}\right\}$, with $\left\|g-g_{m} \mid \mathcal{A}_{u_{2}, p_{2}, q_{2}}^{s_{2}}\left(B_{e}(x, R)\right)\right\|<c \varepsilon$. Then $\left\|f-g_{m}|\Omega| \mathcal{A}_{u_{2}, p_{2}, q_{2}}^{s_{2}}(\Omega)\right\|_{*} \leq\left\|g-g_{m} \mid \mathcal{A}_{u_{2}, p_{2}, q_{2}}^{s_{2}}\left(B_{e}(x, R)\right)\right\| \leq c \varepsilon$. This implies that there exists a positive constant $C$ independent of $k$ such that $e_{k}\left(\mathcal{A}_{u_{1}, p_{1}, q_{1}}^{s_{1}}(\Omega) \hookrightarrow \mathcal{A}_{u_{2}, p_{2}, q_{2}}^{s_{2}}(\Omega)\right) \leq$ $C e_{k}\left(\mathcal{A}_{u_{1}, p_{1}, q_{1}}^{s_{1}}\left(B_{e}(0,1)\right) \hookrightarrow \mathcal{A}_{u_{2}, p_{2}, q_{2}}^{s_{2}}\left(B_{e}(0,1)\right)\right)$.

To prove the opposite inequality we can take a ball $B(x, r)$ such that $\overline{B(x, r)} \subset \Omega$ and argue in a similar way as above.

Now we can concentrate on the entropy numbers of the compact embedding characterised by Theorem 2.10 ,

Preparation. Recall that $\Omega \subset \mathbb{R}^{d}$ is a bounded $C^{\infty}$ domain and the spaces are defined by restriction, see Definition 2.8. Let $\Omega_{t}=\left\{x \in \mathbb{R}^{d}: \operatorname{dist}(x, \Omega)<t\right\}$, for some $t>0$. We choose a dyadic cube $Q$ (applying some appropriate dilations or translations first, if necessary) such that $\operatorname{supp} \psi_{i, \nu, m} \subset Q$ if $\operatorname{supp} \psi_{i, \nu, m} \cap \Omega_{t} \neq \emptyset$, and $\operatorname{supp} \phi_{0, m} \subset Q$ if $\operatorname{supp} \phi_{0, m} \cap \Omega_{t} \neq \emptyset$. Let $h \in C_{0}^{\infty}\left(\mathbb{R}^{d}\right)$ be a test function such that $\operatorname{supp} h \subset \Omega_{t}$ and $h(x)=1$ for any $x \in \Omega_{t / 2}$. We choose $N \in \mathbb{N}$ such that $N^{-1} \leq p_{i} \leq u_{i}, N^{-1}<q_{i}$ and $\left|s_{i}\right|<N, i=1,2$. Then there exists an extension operator ext $N_{N}$, common for both spaces $\mathcal{N}_{u_{1}, p_{1}, q_{1}}^{s_{1}}(\Omega)$ and $\mathcal{N}_{u_{2}, p_{2}, q_{2}}^{s_{2}}(\Omega)$, with re $\circ M_{h}=$ re, that is, re $\circ M_{h} \circ \operatorname{ext}_{N}=$ id on $\mathcal{N}_{u_{i}, p_{i}, q_{i}}^{s_{i}}(\Omega)$, cf. [35]. Here $M_{h}$ is the natural pointwise multiplication mapping $M_{h}: f \ni \mathcal{S}\left(\mathbb{R}^{d}\right) \mapsto h \cdot f$. Now using the wavelet characterisation of the spaces $\mathcal{N}_{u, p, q}^{s}\left(\mathbb{R}^{d}\right)$ by Daubechies wavelets, cf. Theorem 2.5, in particular the isomorphism $T$ defined by [2.6], one can factorise the embeddings for function spaces via the embeddings of the corresponding sequence spaces. More precisely, the following diagram is commutative

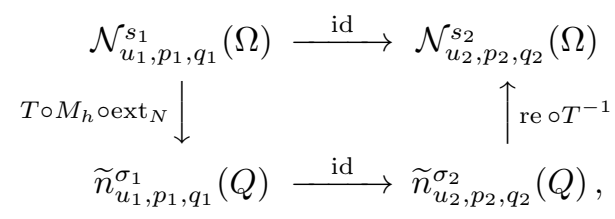

with $\sigma_{i}=s_{i}+\frac{d}{2}$. Thus the multiplicativity of entropy numbers 2.11 immediately yields

$$
e_{k}\left(\mathrm{id}: \mathcal{N}_{u_{1}, p_{1}, q_{1}}^{s_{1}}(\Omega) \rightarrow \mathcal{N}_{u_{2}, p_{2}, q_{2}}^{s_{2}}(\Omega)\right) \leq c e_{k}\left(\mathrm{id}: \widetilde{n}_{u_{1}, p_{1}, q_{1}}^{\sigma_{1}}(Q) \rightarrow \widetilde{n}_{u_{2}, p_{2}, q_{2}}^{\sigma_{2}}(Q)\right)
$$


Conversely, by the diffeomorphic properties of Besov-Morrey spaces, using translations and dilations if necessary we can assume that the domain $\Omega$ satisfies the following conditions: there exists $\nu_{0} \geq 0$ such that $Q_{\nu_{0}, 0} \subset \Omega$, and $\operatorname{supp} \psi_{i, \nu, m} \subset \Omega$ if $Q_{\nu, m} \subset Q_{\nu_{0}, 0}$. Let $\widetilde{T}$ denote the restriction of the isomorphism $T^{-1}$ to the subspaces $n_{u, p, q}^{s}\left(Q_{\nu_{0}, 0}\right) \subset n_{u, p, q}^{s}\left(\mathbb{R}^{d}\right)$, cf. [2.6. Then $\widetilde{T}(\lambda) \in \mathcal{N}_{u, p, q}^{s}\left(\mathbb{R}^{d}\right)$ and $\operatorname{supp} \widetilde{T}(\lambda) \subset \Omega$ for any $\lambda \in n_{u, p, q}^{s}\left(Q_{\nu_{0}, 0}\right)$. Moreover, there exists a positive $\varepsilon>0$ such that for any $\lambda \in n_{u, p, q}^{s}\left(Q_{\nu_{0}, 0}\right), \operatorname{dist}\left(\operatorname{supp} \widetilde{T}(\lambda), \mathbb{R}^{d} \backslash \Omega\right)>\varepsilon$. If we denote by $P: n_{u, p, q}^{s}\left(\mathbb{R}^{d}\right) \rightarrow n_{u, p, q}^{s}\left(Q_{\nu_{0}, 0}\right)$ the usual projection in sequence spaces, then we get the following commutative diagram

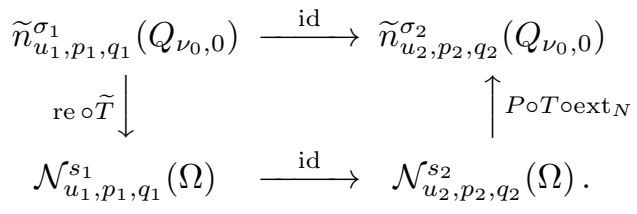

Thus obvious arguments immediately yield

$$
\begin{aligned}
e_{k}\left(\mathrm{id}: \widetilde{n}_{u_{1}, p_{1}, q_{1}}^{\sigma_{1}}(Q) \rightarrow \widetilde{n}_{u_{2}, p_{2}, q_{2}}^{\sigma_{2}}(Q)\right) & \sim e_{k}\left(\mathrm{id}: \widetilde{n}_{u_{1}, p_{1}, q_{1}}^{\sigma_{1}}\left(Q_{\nu_{0}, 0}\right) \rightarrow \widetilde{n}_{u_{2}, p_{2}, q_{2}}^{\sigma_{2}}\left(Q_{\nu_{0}, 0}\right)\right) \\
& \leq c e_{k}\left(\mathrm{id}: \mathcal{N}_{u_{1}, p_{1}, q_{1}}^{s_{1}}(\Omega) \rightarrow \mathcal{N}_{u_{2}, p_{2}, q_{2}}^{s_{2}}(\Omega)\right)
\end{aligned}
$$

Please note that we may take the same system of wavelets, and thus the same operator $\widetilde{T}$, for $\mathcal{N}_{u_{1}, p_{1}, q_{1}}^{s_{1}}\left(\mathbb{R}^{d}\right)$ and $\mathcal{N}_{u_{2}, p_{2}, q_{2}}^{s_{2}}\left(\mathbb{R}^{d}\right)$.

Hence upper and lower estimates for the entropy numbers of sequence space embeddings lead immediately to upper and lower estimates for the corresponding function space embeddings, using the multiplicativity of entropy numbers (2.11) again. So in view of our results in Section 3 and the above arguments we have the following results.

Theorem 4.2. Let $\Omega$ be an arbitrary bounded domain in $\mathbb{R}^{d}$. Let $s_{i} \in \mathbb{R}, 0<q_{i} \leq \infty, 0<p_{i} \leq$ $u_{i}<\infty, i=1,2$. Assume that

(i) either

$$
\frac{s_{1}-s_{2}}{d}>\max \left\{0, \frac{1}{u_{1}}-\frac{1}{u_{2}}, \frac{p_{1}}{u_{1}}\left(\frac{1}{p_{1}}-\frac{1}{p_{2}}\right)\right\}=0,
$$

(ii) or

$$
\frac{s_{1}-s_{2}}{d}>\max \left\{0, \frac{1}{u_{1}}-\frac{1}{u_{2}}, \frac{p_{1}}{u_{1}}\left(\frac{1}{p_{1}}-\frac{1}{p_{2}}\right)\right\}=\frac{1}{u_{1}}-\frac{1}{u_{2}}>0 .
$$

Then

$$
e_{k}\left(\operatorname{id}_{\mathcal{A}}: \mathcal{A}_{u_{1}, p_{1}, q_{1}}^{s_{1}}(\Omega) \hookrightarrow \mathcal{A}_{u_{2}, p_{2}, q_{2}}^{s_{2}}(\Omega)\right) \sim k^{-\frac{s_{1}-s_{2}}{d}} .
$$

Proof. In view of Lemma 4.1 it is sufficient to consider a $C^{\infty}$ domain. In the Besov-Morrey situation, that is, when $\mathcal{A}=\mathcal{N}$, Theorem 3.10 together with our preceding remarks covers the results. Otherwise, when $\mathcal{A}=\mathcal{E}$, then $\left[2.2\right.$ (which remains true in the situation when $\mathbb{R}^{d}$ is replaced by $\Omega$ ) and the independence of all above assumptions of the fine parameters $q_{i}$, $i=1,2$, implies the assertions, where we benefit again from the multiplicativity property of entropy numbers 2.11 .

Remark 4.3. If $u=p$, then $\mathcal{A}_{u, p, q}^{s}(\Omega)=A_{p, q}^{s}(\Omega)$, so we can compare our above result with the well-known situation of the embedding $\operatorname{id}_{A}: A_{p_{1}, q_{1}}^{s_{1}}(\Omega) \rightarrow A_{p_{2}, q_{2}}^{s_{2}}(\Omega)$, where $s_{i} \in \mathbb{R}, 0<p_{i}, q_{i} \leq \infty$ ( $p_{i}<\infty$ in the $F$-case), $i=1,2$, and the spaces $A_{p, q}^{s}(\Omega)$ are defined by restriction. Then $\operatorname{id}_{A}$ is compact, if, and only if, $\frac{s_{1}-s_{2}}{d}>\max \left\{0, \frac{1}{p_{1}}-\frac{1}{p_{2}}\right\}$, recall also 2.10 . Note that in this situation the above cases (i) and (ii) of Theorem 4.2 cover all possible cases of compactness already. Edmunds and Triebel proved in [6,7] (see also [8, Thm. 3.3.3/2]) that

$$
e_{k}\left(\operatorname{id}_{A}: A_{p_{1}, q_{1}}^{s_{1}}(\Omega) \hookrightarrow A_{p_{2}, q_{2}}^{s_{2}}(\Omega)\right) \sim k^{-\frac{s_{1}-s_{2}}{d}}, \quad k \in \mathbb{N},
$$

which perfectly coincides with our findings 4.3.

We return to the non-classical case, that is, when $p_{1}<u_{1}$ or $p_{2}<u_{2}$. 
Theorem 4.4. Let $\Omega$ be an arbitrary bounded domain in $\mathbb{R}^{d}$. Let $s_{i} \in \mathbb{R}, 0<q_{i} \leq \infty, 0<p_{i} \leq$ $u_{i}<\infty, i=1,2$, and

$$
\frac{s_{1}-s_{2}}{d}>\frac{p_{1}}{u_{1}}\left(\frac{1}{p_{1}}-\frac{1}{p_{2}}\right)>\max \left\{0, \frac{1}{u_{1}}-\frac{1}{u_{2}}\right\} .
$$

(i) If $\frac{s_{1}-s_{2}}{d}>\frac{1}{p_{1}}-\frac{1}{p_{2}}$, then

$$
e_{k}\left(\operatorname{id}_{\mathcal{A}}: \mathcal{A}_{u_{1}, p_{1}, q_{1}}^{s_{1}}(\Omega) \hookrightarrow \mathcal{A}_{u_{2}, p_{2}, q_{2}}^{s_{2}}(\Omega)\right) \sim k^{-\frac{s_{1}-s_{2}}{d}}
$$

(ii) If $\frac{p_{1}}{u_{1}}\left(\frac{1}{p_{1}}-\frac{1}{p_{2}}\right)<\frac{s_{1}-s_{2}}{d} \leq \frac{1}{p_{1}}-\frac{1}{p_{2}}$, then there exists some $c>0$ and for any $\varepsilon>0$ some $c_{\varepsilon}>0$ such that for all $k \in \mathbb{N}$,

$$
c k^{-\frac{u_{1}}{u_{1}-p_{1}}\left(\frac{s_{1}-s_{2}}{d}-\frac{p_{1}}{u_{1}}\left(\frac{1}{p_{1}}-\frac{1}{p_{2}}\right)\right)} \leq e_{k}\left(\operatorname{id}_{\mathcal{A}}\right) \leq c_{\varepsilon} k^{-\frac{u_{1}}{u_{1}-p_{1}}\left(\frac{s_{1}-s_{2}}{d}-\frac{p_{1}}{u_{1}}\left(\frac{1}{p_{1}}-\frac{1}{p_{2}}\right)\right)+\varepsilon} .
$$

Proof. We follow the same strategy as in case of Theorem 4.2, using this time Theorem 3.13 ,

Remark 4.5. We think that the outcome is really remarkable for at least two reasons: although the result in (ii) is not yet sharp, it shows that the 'classical' asymptotic behaviour of the entropy numbers, $e_{k} \sim k^{-\frac{s_{1}-s_{2}}{d}}$, cannot be true in this case. This is indeed surprising and was not to be expected before. Secondly, we want to point out again the interplay between the Morrey fine parameters $p_{i}$ and the smoothness parameters $s_{i}$ in this case.

We briefly return to our remark at the end of Section 3 concerning the situation $s_{1}-s_{2}=$ $d\left(\frac{1}{p_{1}}-\frac{1}{p_{2}}\right)$ where the above cases (i) and (ii) of Theorem 4.4 meet and we obtain the two-sided estimate

$$
c k^{-\frac{s_{1}-s_{2}}{d}} \leq e_{k}\left(\operatorname{id}_{\mathcal{A}}: \mathcal{A}_{u_{1}, p_{1}, q_{1}}^{s_{1}}(\Omega) \hookrightarrow \mathcal{A}_{u_{2}, p_{2}, q_{2}}^{s_{2}}(\Omega)\right) \leq c_{\varepsilon} k^{-\frac{s_{1}-s_{2}}{d}+\varepsilon} .
$$

In similar limiting cases for embeddings an additional log-term appears occasionally, and even an influence of the parameters $q_{i}, i=1,2$, and a different behaviour for the case $\mathcal{A}=\mathcal{N}$ or $\mathcal{A}=\mathcal{E}$ has been found, see [10, 23, 24]. But we have no claim for our limiting situation at the moment. Moreover, we have observed a different phenomenon in [11 concerning some weighted situation: in that case the local behaviour of the weight was involved in the characterisation of the compactness of that embedding, while the entropy numbers 'ignored' this local part completely. So it remains a tricky business after all with many open questions.

Remark 4.6. Note that Theorems 4.2 and 4.4 imply, in particular, the extension of Theorem 2.10 to arbitrary bounded domains in view of the properties of entropy numbers.

We consider some special cases, and begin with the case $p_{1}=u_{1}$, that is, when the source space $\mathcal{A}_{u_{1}, p_{1}, q_{1}}^{s_{1}}(\Omega)$ coincides with the space $A_{p_{1}, q_{1}}^{s_{1}}(\Omega)$. Then Theorem 4.4, i.e. 4.4 is impossible and we arrive in all possible settings at the classical behaviour for the entropy numbers, recall Remark 4.3.

Corollary 4.7. Let $\Omega$ be a bounded domain in $\mathbb{R}^{d}, s_{i} \in \mathbb{R}, 0<q_{i} \leq \infty, 0<p_{i} \leq u_{i}<\infty, i=1,2$, and (2.10) satisfied. Assume $p_{1}=u_{1}$. Then

$$
e_{k}\left(\operatorname{id}: A_{p_{1}, q_{1}}^{s_{1}}(\Omega) \hookrightarrow \mathcal{A}_{u_{2}, p_{2}, q_{2}}^{s_{2}}(\Omega)\right) \sim e_{k}\left(\operatorname{id}_{A}: A_{p_{1}, q_{1}}^{s_{1}}(\Omega) \hookrightarrow A_{p_{2}, q_{2}}^{s_{2}}(\Omega)\right) \sim k^{-\frac{s_{1}-s_{2}}{d}}
$$

Proof. This follows immediately from Theorem 4.2 when $p_{1}=u_{1}$.

Remark 4.8. This 'negligence' of the (larger) Morrey target space $\mathcal{A}_{u_{2}, p_{2}, q_{2}}^{s_{2}}(\Omega)$ (compared with $A_{p_{2}, q_{2}}^{s_{2}}(\Omega)$ ) is not completely new: it has its counterpart in the related compactness result [13, Cor. 4.2]. Thus the really new and surprising asymptotic behaviour 4.6) of $e_{k}\left(\mathrm{id}_{\mathcal{A}}\right)$ can only appear when $p_{1}<u_{1}$.

We finally consider the case $p_{2}=u_{2}$ and may also cover the $L_{r}$ scale, including the case $r=\infty$ described in Proposition 2.11. In that situation case (ii) of Theorem 4.2 is excluded and 2.10) reads as

$$
\frac{s_{1}-s_{2}}{d}>\max \left\{0, \frac{p_{1}}{u_{1}}\left(\frac{1}{p_{1}}-\frac{1}{p_{2}}\right)\right\}=\frac{p_{1}}{u_{1}}\left(\frac{1}{p_{1}}-\frac{1}{p_{2}}\right)_{+} .
$$


Corollary 4.9. Let $\Omega$ be a bounded domain in $\mathbb{R}^{d}, s_{i} \in \mathbb{R}, 0<q_{i} \leq \infty, 0<p_{i} \leq u_{i}<\infty, i=1,2$, and 4.7] satisfied. Assume that $p_{2}=u_{2}$.

(i) If $\frac{s_{1}-s_{2}}{d}>\left(\frac{1}{p_{1}}-\frac{1}{p_{2}}\right)_{+}$, then

$$
e_{k}\left(\operatorname{id}: \mathcal{A}_{u_{1}, p_{1}, q_{1}}^{s_{1}}(\Omega) \hookrightarrow A_{p_{2}, q_{2}}^{s_{2}}(\Omega)\right) \sim k^{-\frac{s_{1}-s_{2}}{d}}
$$

(ii) If $\frac{p_{1}}{u_{1}}\left(\frac{1}{p_{1}}-\frac{1}{p_{2}}\right)<\frac{s_{1}-s_{2}}{d} \leq \frac{1}{p_{1}}-\frac{1}{p_{2}}$, then there exists some $c>0$ and for any $\varepsilon>0$ some $c_{\varepsilon}>0$ such that for all $k \in \mathbb{N}$,

$$
c k^{-\frac{u_{1}}{u_{1}-p_{1}}\left(\frac{s_{1}-s_{2}}{d}-\frac{p_{1}}{u_{1}}\left(\frac{1}{p_{1}}-\frac{1}{p_{2}}\right)\right)} \leq e_{k}(\text { id }) \leq c_{\varepsilon} k^{-\frac{u_{1}}{u_{1}-p_{1}}\left(\frac{s_{1}-s_{2}}{d}-\frac{p_{1}}{u_{1}}\left(\frac{1}{p_{1}}-\frac{1}{p_{2}}\right)\right)+\varepsilon} .
$$

Proof. This follows from Theorems 4.2 and 4.4 with $p_{2}=u_{2}$.

Corollary 4.10. Let $\Omega$ be a bounded domain in $\mathbb{R}^{d}, 1 \leq r \leq \infty, 0<p \leq u<\infty$, and $s>$ $d \frac{p}{u}\left(\frac{1}{p}-\frac{1}{r}\right)_{+}$.

(i) If $p \geq r$, then

$$
e_{k}\left(\text { id }: \mathcal{A}_{u, p, q}^{s}(\Omega) \hookrightarrow L_{r}(\Omega)\right) \sim k^{-\frac{s}{d}}
$$

(ii) If $p<r$ and $d \frac{p}{u}\left(\frac{1}{p}-\frac{1}{r}\right)<s \leq d\left(\frac{1}{p}-\frac{1}{r}\right)$, then there exists some $c>0$ and for any $\varepsilon>0$ some $c_{\varepsilon}>0$ such that for all $k \in \mathbb{N}$,

$$
c k^{-\frac{u}{u-p}\left(\frac{s}{d}-\frac{p}{u}\left(\frac{1}{p}-\frac{1}{r}\right)\right)} \leq e_{k}\left(\text { id }: \mathcal{A}_{u, p, q}^{s}(\Omega) \hookrightarrow L_{r}(\Omega)\right) \leq c_{\varepsilon} k^{-\frac{u}{u-p}\left(\frac{s}{d}-\frac{p}{u}\left(\frac{1}{p}-\frac{1}{r}\right)\right)+\varepsilon},
$$

where $L_{\infty}(\Omega)$ can be replaced in all cases by $C(\Omega)$.

Proof. The result follows from Corollary 4.9 together with the well-known embeddings $B_{r, 1}^{0}(\Omega) \hookrightarrow L_{r}(\Omega) \hookrightarrow B_{r, \infty}^{0}(\Omega)$ and the multiplicativity of entropy numbers 2.11 .

Remark 4.11. We obtained in [16,50] some first results for approximation numbers of the embedding $\operatorname{id}_{\mathcal{A}}$. In this context we refer to [1, Sect. 6] where also the periodic case and more general Morrey type spaces are studied.

Remark 4.12. Thanks to one reviewer we were pointed to the recent preprint [25] which is based on [5]. It might well be that using this approach one could circumvent our above interpolation argument and thus seal the $\varepsilon$-gap in the exponents in Theorems 3.13 (ii) and 4.4(ii), respectively. We leave it as an open question here.

\section{Acknowledgment}

We are indebted to the referees of the first version of that paper for their valuable remarks which helped to improve the results and the presentation.

\section{References}

[1] Z. Baituyakova and W. Sickel. Strong summability of Fourier series and generalized Morrey spaces. Anal. Math., 43(3):371-414, 2017.

[2] B. Carl, Entropy numbers, s-numbers, and Eigenvalue Problems, Journal of Functional Analysis 41(1981), 290-306.

[3] B. Carl and I. Stephani. Entropy, compactness and the approximation of operators. Cambridge Univ. Press, Cambridge, 1990.

[4] D.E. Edmunds and W.D. Evans. Spectral Theory and Differential Operators. Clarendon Press, Oxford, 1987. 
[5] D. E. Edmunds and Yu. Netrusov. Schütt's theorem for vector-valued sequence spaces. J. Approx. Theory, 178:13-21, 2014.

[6] D.E. Edmunds and H. Triebel, Entropy numbers and approximation numbers in function spaces. Proc. London Math. Soc., 58(3):137-152, 1989.

[7] D.E. Edmunds and H. Triebel, Entropy numbers and approximation numbers in function spaces II. Proc. London Math. Soc., 64(3):153-169, 1992.

[8] D.E. Edmunds and H. Triebel. Function spaces, entropy numbers, differential operators. Cambridge Univ. Press, Cambridge, 1996.

[9] D.D. Haroske, S.D. Moura, and L. Skrzypczak, Smoothness Morrey Spaces of regular distributions, and some unboundedness properties. Nonlinear Analysis Series A: Theory, Methods and Applications, 139 (2016), 218-244.

[10] D.D. Haroske and L. Skrzypczak, Entropy and approximation numbers of embeddings of function spaces with Muckenhoupt weights, I, Rev. Mat. Complut. 21 (2008), 135-177.

[11] D.D. Haroske and L. Skrzypczak. Entropy and approximation numbers of embeddings of function spaces with Muckenhoupt weights, II. General weights. Ann. Acad. Sci. Fenn. Math., 36:111-138, 2011.

[12] D.D. Haroske and L. Skrzypczak, Continuous embeddings of Besov-Morrey function spaces, Acta Math. Sin. (Engl. Ser.) 28 (2012), 1307-1328.

[13] D.D. Haroske and L. Skrzypczak, Embeddings of Besov-Morrey spaces on bounded domains, Studia Math. 218 (2013), 119-144.

[14] D.D. Haroske and L. Skrzypczak, On Sobolev and Franke-Jawerth embeddings of smoothness Morrey spaces. Rev. Mat. Complut. 27 (2014), 541-573.

[15] D.D. Haroske and L. Skrzypczak. Morrey Sequence Spaces: Pitt's Theorem and compact embeddings. Constr. Approx., accepted.

[16] D.D. Haroske and L. Skrzypczak. Some quantitative result on compact embeddings in smoothness Morrey spaces on bounded domains; an approach via interpolation. submitted

[17] D. Haroske and H. Triebel, Entropy numbers in weighted function spaces and eigenvalue distributions of some degenerate pseudodifferential operators I, Math. Nachr. 167, 131156 (1994).

[18] H. König, Eigenvalue distribution of compact operators. Birkhäuser, Basel, 1986.

[19] H. Kozono and M. Yamazaki, Semilinear heat equations and the Navier-Stokes equation with distributions in new function spaces as initial data. Comm. Partial Differential Equations 19 (1994), 959-1014.

[20] Th. Kühn. A lower estimate for entropy numbers. J. Approx. Theory, 110:120-124, 2001.

[21] Th. Kühn. Compact embeddings of Besov spaces in exponential Orlicz spaces. J. London Math. Soc. (2), 67(1):235-244, 2003.

[22] Th. Kühn, H.-G. Leopold, W. Sickel, and L. Skrzypczak. Entropy numbers of Sobolev embeddings of radial Besov spaces. J. Approx. Theory, 121:244-268, 2003.

[23] Th. Kühn, H.-G. Leopold, W. Sickel and L. Skrzypczak, Entropy numbers of embeddings of weighted Besov spaces. Constr. Approx. 23 (2006), 61-77.

[24] H.-G. Leopold. Embeddings and entropy numbers in Besov spaces of generalized smoothness. In H. Hudzik and L. Skrzypczak, editors, Function spaces, pages 323-336. The fifth conference, Lecture notes in pure and applied math. 213, Marcel Dekker, 2000. 
[25] S. Mayer and T. Ullrich. Entropy numbers of finite dimensional mixed-norm balls and function space embeddings with small mixed smoothness. Preprint, arXiv: 1904.04619.

[26] A.L. Mazzucato, Besov-Morrey spaces: function space theory and applications to non-linear PDE. Trans. Amer. Math. Soc. 355 (2003), 1297-1364.

[27] C.B. Morrey, On the solutions of quasi-linear elliptic partial differential equations. Trans. Amer. Math. Soc. 43 (1938), 126-166.

[28] J. Peetre, On the theory of $\mathcal{L}_{p, \lambda}$ spaces. J. Funct. Anal. 4 (1969), 71-87.

[29] A. Pietsch. Operator Ideals. North-Holland, Amsterdam, 1980.

[30] A. Pietsch. Eigenvalues and s-Numbers. Akad. Verlagsgesellschaft Geest \& Portig, Leipzig, 1987.

[31] G. Pisier: The volume of convex bodies and Banach space geometry. Cambridge University Press , Cambridge, 1989.

[32] M. Rosenthal, Local means, wavelet bases, representations, and isomorphisms in BesovMorrey and Triebel-Lizorkin-Morrey spaces. Math. Nachr. 286 (2013), 59-87.

[33] Y. Sawano, A note on Besov-Morrey spaces and Triebel-Lizorkin-Morrey spaces. Acta Math. Sin. (Engl. Ser.) 25 (2009), 1223-1242.

[34] Y. Sawano, Wavelet characterizations of Besov-Morrey and Triebel-Lizorkin-Morrey spaces, Funct. Approx. Comment. Math., 38 (2008), 93-107.

[35] Y. Sawano, Besov-Morrey spaces and Triebel-Lizorkin-Morrey spaces on domains, Math. Nachr. 283 (2010), no. 10, 1456-1487.

[36] Y. Sawano and H. Tanaka, Decompositions of Besov-Morrey spaces and Triebel-LizorkinMorrey spaces. Math. Z. 257 (2007), 871-905.

[37] Y. Sawano and H. Tanaka, Besov-Morrey spaces and Triebel-Lizorkin-Morrey spaces for non-doubling measures. Math. Nachr. 282 (2009), 1788-1810.

[38] C. Schütt, Entropy numbers of diagonal operators between symmetric Banach spaces. J. Approx. Theory 40 (1984), 121-128.

[39] W. Sickel, Smoothness spaces related to Morrey spaces - a survey. I. Eurasian Math. J. 3 (2012), 110-149.

[40] W. Sickel, Smoothness spaces related to Morrey spaces - a survey. II, Eurasian Math. J. 4 (2013), 82-124.

[41] L. Tang and J. Xu, Some properties of Morrey type Besov-Triebel spaces. Math. Nachr. 278 (2005), 904-917.

[42] H. Triebel. Interpolation theory, function spaces, differential operators. North-Holland, Amsterdam, 1978.

[43] H. Triebel, Theory of Function Spaces. Birkhäuser, Basel, 1983.

[44] H. Triebel, Theory of Function Spaces II. Birkhäuser, Basel, 1992.

[45] H. Triebel. Fractals and spectra. Birkhäuser, Basel, 1997.

[46] H. Triebel, Theory of function spaces III, Birkhäuser, Basel, 2006.

[47] H. Triebel, Local Function Spaces, Heat and Navier-Stokes Equations. EMS Tracts in Mathematics 20, European Mathematical Society (EMS), Zürich, 2013.

[48] H. Triebel, Hybrid Function Spaces, Heat and Navier-Stokes Equations. EMS Tracts in Mathematics 24, European Mathematical Society (EMS), Zürich, 2015. 
[49] W. Yuan, D.D. Haroske, L. Skrzypczak, and D. Yang, Embedding properties of Besov-type spaces. Appl. Anal. 94 (2015), no. 2, 318-340.

[50] W. Yuan, D.D. Haroske, S.D. Moura, L. Skrzypczak, and D. Yang, Limiting embeddings in smoothness Morrey spaces, continuity envelopes and applications. J. Approx. Theory, 192 (2015) 306-335.

[51] W. Yuan, W. Sickel, and D. Yang, Morrey and Campanato Meet Besov, Lizorkin and Triebel. Lecture Notes in Mathematics 2005, Springer-Verlag, Berlin, 2010. 